\title{
CONTROLLABILITY PROPERTIES OF A CLASS OF SYSTEMS MODELING SWIMMING MICROSCOPIC ORGANISMS
}

\author{
Mario Sigalotti $^{1,2}$ And Jean-Claude Vivalda ${ }^{2,3}$
}

\begin{abstract}
We consider a finite-dimensional model for the motion of microscopic organisms whose propulsion exploits the action of a layer of cilia covering its surface. The model couples Newton's laws driving the organism, considered as a rigid body, with Stokes equations governing the surrounding fluid. The action of the cilia is described by a set of controlled velocity fields on the surface of the organism. The first contribution of the paper is the proof that such a system is generically controllable when the space of controlled velocity fields is at least three-dimensional. We also provide a complete characterization of controllable systems in the case in which the organism has a spherical shape. Finally, we offer a complete picture of controllable and non-controllable systems under the additional hypothesis that the organism and the fluid have densities of the same order of magnitude.
\end{abstract}

Mathematics Subject Classification. 37C20, 70Q05, 76Z10, 93B05, 93C10.

Received November 15, 2007. Revised January 4, 2009.

Published online August 11, 2009.

\section{INTRODUCTION}

The mathematical description of the motion of swimming organisms and of the mechanisms of their propulsion is a challenging and wide field of research. This paper aims at giving a contribution to such a domain in a comparatively narrow direction.

The object of our study are microscopic organisms. The most prominent aspect of the models describing their motion is the very high level of viscosity $[3,18,22]$. The mechanism of their propulsion depends heavily on the different species and admits a great variety of models (see, for instance, $[2,7,9,17]$ and references therein).

Among microscopic organisms we will further restrict our attention to the class of ciliata, whose propulsion is determined by cilia, hair-like organelles covering their surface and whose size is very small compared with that of the organism (see [4,5,9]). Recently San Martín et al. [19] proposed a control theory approach to the description of ciliata. Following the former literature (and in particular $[9,16]$ ) they assume that the organism is a rigid body and that the motion of the cilia is described by a set of controlled velocity fields on a surface

\footnotetext{
Keywords and phrases. Swimming micro-organisms, ciliata, high viscosity, nonlinear systems, controllability.

${ }^{1}$ Institut Élie Cartan de Nancy, UMR 7502 INRIA/Nancy-Université/CNRS, B.P. 239, 54506 Vandouvre-lès-Nancy Cedex,

France. mario.sigalotti@inria.fr

2 Équipe-projet CORIDA, INRIA Nancy - Grand Est, France.

3 Laboratoire et Département de Mathématiques, UMR 7122 Université de Metz/CNRS, Bât. A, Île du Saulcy, 57045 Metz Cedex 1, France.
} 
enclosing the layer of cilia. They are therefore faced to an infinite-dimensional control system coupling NavierStokes equations, which describe the motion of the fluid, and the equations for the rigid body arising form Newton's laws. The hypothesis of high viscosity (i.e., of low Reynolds number) mentioned above is exploited to reduce such a control system to a finite-dimensional version of it. The state-space is given by the coordinates of the center of mass of the organism, its orientation and its angular and linear velocities.

Summarizing, San Martín et al. single out a 12-dimensional nonlinear control system which is affine in the controls. The control system depends on a number of parameters which are explicitly derived as functions of the shape of the organism, of its mass distribution, and of the surface velocity fields describing the motion of the cilia. The authors study the control properties of the system proving that it is generically controllable when the number of controlled vector fields is at least six (see Sect. 5 for details). They also provide some example of controllable systems in the case in which the organism has a spherical shape. In this special case they also study the controllability properties of the linearization of the system at its rest position.

Our scope is to enhance the results of [19] basically in three directions: firstly we want to give sharper results on the generic controllability of the system; secondly we want to obtain a complete characterization of controllable systems in the case in which the organism is spherical; thirdly we want to provide a complete picture of controllable and non-controllable systems in the simplified situation which - following again [19] comes into effect in the case in which the organism and the fluid have densities of the same order of magnitude. The overall aim is to provide a better understanding of the dependence of the controllability properties of the model proposed in [19] on the physical quantities characterizing it. The hard aspect of the model is indeed the difficulty to obtain reliable expressions for the surface velocity fields describing the motions of the cilia. In this regard, it is important to provide as precise as possible parametric analysis of the controllability properties of the system.

The paper is organized as follows. Section 2 introduces the control theoretical language adopted throughout the paper. Section 3 presents the control system obtained in [19] and recalls the relations between the physical quantities characterizing the organism and the parameters appearing in the model. Section 4 is technical and contains an abstract controllability result used in the later sections. Section 5 is devoted to the analysis of the genericity of the controllability of the system introduced in Section 3. Genericity is initially formulated in terms of the parameters appearing in the finite-dimensional system and then expressed in terms of the physical objects they depend on (Sect. 5.3). The main result is that the system is generically controllable if the number of controlled vector fields is at least three and that one controlled vector field suffices for its generic accessibility (Thm. 5.1). Section 6 analyzes the case in which the organism is spherical and provides a complete characterization of the sets of parameters that make the system controllable (Thm. 6.1). Finally, Section 7 establishes a characterization of controllable systems when the organism can be assumed to have the same density as the fluid (Thm. 7.2). The result follows from a further reduction of the system that transforms it into a six-dimensional one. The reduction procedure, suggested in [19], is carried out in full details.

\section{Notations AND DEFINITIONS}

Let us first introduce some notations: in the following Id denotes the $3 \times 3$ identity matrix, $0_{m \times n}$ is the zero $m \times n$ matrix, $0_{m}=0_{m \times 1}$ is the zero vector in $\mathbf{R}^{m}$. We denote by $\mathscr{M}_{m \times n}$ the set of $m \times n$ real matrices. The interior of a set $W$ is denoted by $\operatorname{Int}(W)$ and its closure by $\operatorname{Clos}(W)$. For simplicity of notation, when no confusion is possible, row and column vectors are identified. The canonical orthonormal basis of $\mathbf{R}^{3}$ is denoted by $\left\{e_{1}, e_{2}, e_{3}\right\}$. For every $\omega \in \mathbf{R}^{3}$ we denote by $S(\omega)$ the skew symmetric matrix such that $S(\omega) z=\omega \times z$ for every $z \in \mathbf{R}^{3}$, that is,

$$
S(\omega)=\left(\begin{array}{ccc}
0 & -\omega_{3} & \omega_{2} \\
\omega_{3} & 0 & -\omega_{1} \\
-\omega_{2} & \omega_{1} & 0
\end{array}\right) .
$$

In what follows we deal with control systems of the type

$$
\dot{q}=f(q, u), \quad q \in M, \quad u \in \mathbf{R}^{m},
$$


where $M$ is a smooth $\left(\mathcal{C}^{\infty}\right)$ manifold and $f: M \times \mathbf{R}^{m} \rightarrow T M$ is smooth with respect to both variables. Moreover, for every $T \geq 0$ the endpoint mapping $E_{T}$ which associates to an initial condition $q_{0} \in M$ and a control function $u \in L^{\infty}\left([0, T], \mathbf{R}^{m}\right)$ the final point of the corresponding trajectory of $(2.1)$ is well defined and continuous on $M \times L^{\infty}\left([0, T], \mathbf{R}^{m}\right)$, where the $L^{1}$ norm is chosen on $L^{\infty}\left([0, T], \mathbf{R}^{m}\right)$. (For these and finer properties of the endpoint mapping see, for instance, [1].)

Let $\mathscr{F}=\left\{f(\cdot, u) \mid u \in \mathbf{R}^{m}\right\}$ be the family of vector fields characterizing $(2.1)$. For every $X=f(\cdot, u) \in \mathscr{F}$ we denote by $\mathrm{e}^{t X}$ the one-parameter group generated by $X$, that is, $\mathrm{e}^{t X}(q)=E_{t}\left(q, u 1_{[0, t]}\right)$. The attainable set from $q \in M$ is the set

$$
\mathcal{A}(q, \mathscr{F})=\cup_{T \geq 0} E_{T}\left(q, L^{\infty}\left([0, T], \mathbf{R}^{m}\right)\right) .
$$

When no confusion is possible we write $\mathcal{A}(q)=\mathcal{A}(q, \mathscr{F})$. If $q^{\prime}$ belongs to $\mathcal{A}(q, \mathscr{F})$, then we say that $q^{\prime}$ is reachable form $q$ by $\mathscr{F}$.

We say that (2.1) (or, equivalently, $\mathscr{F}$ ) is controllable if for every $q \in M$ the set $\mathcal{A}(q)$ is equal to $M$. We say that (2.1) is approximatively controllable if $\mathcal{A}(q)$ is dense in $M$.

Give two vector fields $X$ and $Y$ on $M$, the Lie bracket between $X$ and $Y$ is defined, in a local system of coordinates, by the relation

$$
[X, Y]=(D Y) X-(D X) Y
$$

where $D X$ denotes the derivative of $X$. Denote by $\mathscr{L}$ the Lie algebra generated by $\mathscr{F}$. System $(2.1)$ is Liebracket generating if for every $q \in M$ the set $\mathscr{L}(q)=\{V(q) \mid V \in \mathscr{L}\}$ is equal to $T_{q} M$. Equivalently, we say that the rank of $\mathscr{L}$ is maximal at every $q \in M$.

\section{ThE DYNAMICS OF SWIMMING MICROSCOPIC ORGANISMS}

In [19] the authors describe the swimming of a microscopic organism immersed in an infinite volume of fluid by coupling the dynamics of a rigid body (representing the organism) with the Navier-Stokes equations describing the behavior of the fluid outside the body. Exploiting the hypothesis of very low Reynolds number, they derive a simplified version of the coupled system. The system obtained in this way is finite-dimensional and nonlinear; more precisely, it has the following expression:

$$
\begin{aligned}
\dot{z} & =A z+E(z)+B u \\
\dot{\zeta} & =R \xi \\
\dot{R} & =R S(\omega)
\end{aligned}
$$

where $z=(\xi, \omega) \in \mathbf{R}^{3} \times \mathbf{R}^{3}, \zeta \in \mathbf{R}^{3}, R \in \mathrm{SO}(3)$, and

$$
E(z)=\left(\begin{array}{c}
\xi \times \omega \\
J^{-1}((J \omega) \times \omega)
\end{array}\right) .
$$

Here $J \in \mathscr{M}_{3 \times 3}$ denotes the inertia matrix of the rigid body representing the organism. Recall that $J$ is symmetric and positive definite. The matrix $A \in \mathscr{M}_{6 \times 6}$ is a function of the rigid body and the viscosity of the fluid and its expression is given at the end of the section.

The control function $u$ takes values in $\mathbf{R}^{m}, m \geq 1$, and $B$ is a 6 -by- $m$ matrix, which we write as

$$
B=\left(\begin{array}{c}
B_{1} \\
B_{2}
\end{array}\right)
$$

where both $B_{1}$ and $B_{2}$ belong to $\mathscr{M}_{3 \times m}$.

An important feature of the system above is that (3.1) is a well-defined control system on $\mathbf{R}^{6}$, since it does not depend on $\zeta$ nor $R$. 
Denote by $X_{0}$ the vector field on $\mathbf{R}^{6} \times \mathbf{R}^{3} \times \mathrm{SO}(3)$ which is the drift of the control system (3.1)-(3.3), that is,

$$
X_{0}(z, \zeta, R)=\left(\begin{array}{c}
A z+E(z) \\
R \xi \\
R S(\omega)
\end{array}\right)
$$

Let moreover $X_{1}, \ldots, X_{m}$ be the controlled vector fields, so that system (3.1)-(3.3) can be written as

$$
\dot{q}=X_{0}(q)+\sum_{i=1}^{m} u_{i} X_{i}(q), \quad q \in \mathbf{R}^{9} \times \mathrm{SO}(3) .
$$

Define $\mathscr{L}$ as the Lie algebra generated by $X_{0}, X_{1}, \ldots, X_{m}$.

We say that a vector field $X$ on $\mathbf{R}^{9} \times \mathrm{SO}(3)$ is a constant vector field if there exist $v_{1}, v_{2} \in \mathbf{R}^{3}$ such that $X:(\xi, \omega, \zeta, R) \mapsto\left(v_{1}, v_{2}, 0_{6}\right)$ and we write $X=\left(v_{1}, v_{2}, 0_{6}\right)$. Clearly, $X_{1}, \ldots, X_{m}$ are constant vector fields. Notice that if $V=\left(v_{1}, v_{2}, 0_{6}\right)$ and $W=\left(w_{1}, w_{2}, 0_{6}\right)$ are constant vector fields, then

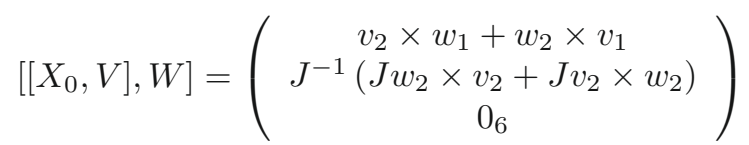

is a constant vector field as well.

Remark 3.1. For every constant vector field $V=\left(v_{1}, v_{2}, 0_{6}\right)$, the bracket $\left[X_{0}, V\right]$ is of the form

$$
\left[X_{0}, V\right](z, \zeta, R)=\left(G(z, V),-R v_{1},-R S\left(v_{2}\right)\right)
$$

for some smooth map $G$ with values in $\mathbf{R}^{6}$. Therefore, if the constant vector fields belonging to $\mathscr{L}$ span $\mathbf{R}^{6} \times\left\{0_{6}\right\}$, then system (3.1)-(3.3) is Lie-bracket generating.

We conclude the section by recalling the analytic characterization of the coefficients appearing in $A, B$ and $J$. Let $\Omega \subset \mathbf{R}^{3}$ be an open bounded connected set whose boundary is of class $\mathcal{C}^{2}$, representing the (rigid) shape of the organism. Then the coefficients $J_{i j}$ of $J$ are given by

$$
J_{i j}=\int_{\Omega} \delta(x)\left(e_{i} \times(x-\bar{\xi})\right) \cdot\left(e_{j} \times(x-\bar{\xi})\right) \mathrm{d} x,
$$

where $\bar{\xi}$ denotes the mass center of $\Omega$ and $\delta: \Omega \rightarrow(0, \infty)$ is the (possibly non-constant) density function.

Denote by $F$ the set $\mathbf{R}^{3} \backslash \operatorname{Clos}(\Omega)$ and, for $l \in \mathbf{N}, 1 \leq q \leq \infty$, let

$$
D^{l, q}(F)=\left\{\varphi \in L_{\text {loc }}^{1} \mid \partial^{\alpha} \varphi \in L^{q}(F) \text { for all } \alpha \in \mathbf{N}^{3},|\alpha|=l\right\} .
$$

According to [19], Lemma 3.1, for every $i \in\{1,2,3\}$ there exists a unique solution $\left(h^{(i)}, p^{(i)}\right)$ of

$$
\left\{\begin{aligned}
-\Delta h^{(i)}+\nabla p^{(i)} & =0 & & \text { on } F \\
\operatorname{div} h^{(i)} & =0 & & \text { on } F \\
h^{(i)} & =e_{i} & & \text { on } \partial \Omega \\
\lim _{|y| \rightarrow \infty} h^{(i)}(y) & =0 & &
\end{aligned}\right.
$$

and a unique solution $\left(H^{(i)}, P^{(i)}\right)$ of

$$
\left\{\begin{aligned}
-\Delta H^{(i)}+\nabla P^{(i)} & =0 & & \text { on } F \\
\operatorname{div} H^{(i)} & =0 & & \text { on } F \\
H^{(i)}(y) & =e_{i} \times y & & \text { on } \partial \Omega \\
\lim _{|y| \rightarrow \infty} H^{(i)}(y) & =0 & &
\end{aligned}\right.
$$


such that $h^{(i)}, H^{(i)} \in L^{s}(F) \cap D^{1, r}(F) \cap D^{2, \theta}(F) \cap \mathcal{C}^{\infty}(F)$ and $p^{(i)}, P^{(i)} \in L^{r}(F) \cap D^{1, \theta}(F) \cap \mathcal{C}^{\infty}(F)$ for $s \in(3, \infty]$, $r \in(3 / 2, \infty]$ and $\theta \in(1, \infty)$.

The Cauchy stress is the tensor $\sigma$ defined by the relation

$$
\sigma(v, p)=-p \operatorname{Id}+\mu\left(\frac{\partial v_{k}}{\partial y_{l}}+\frac{\partial v_{l}}{\partial y_{k}}\right)_{1 \leq l, k \leq 3}
$$

where $\mu$ is the Reynolds number of the fluid. For $i=1,2,3$, define

$$
g^{(i)}=\left.\sigma\left(h^{(i)}, p^{(i)}\right) n\right|_{\partial \Omega}, \quad G^{(i)}=\left.\sigma\left(H^{(i)}, P^{(i)}\right) n\right|_{\partial \Omega},
$$

where $n$ is the unit inner normal to $\partial \Omega$.

Denote by $\Theta^{1}, \Theta^{2}, \Upsilon^{1}, \Upsilon^{2}$ the matrices in $\mathscr{M}_{3 \times 3}$ defined by

$$
\begin{array}{ll}
\Theta_{i j}^{1}=-\int_{\partial \Omega} g_{j}^{(i)} \mathrm{d} s, & \Theta_{i j}^{2}=-\int_{\partial \Omega}\left(x \times g^{(i)}\right)_{j} \mathrm{~d} s, \\
\Upsilon_{i j}^{1}=-\int_{\partial \Omega} G_{j}^{(i)} \mathrm{d} s, & \Upsilon_{i j}^{2}=-\int_{\partial \Omega}\left(x \times G^{(i)}\right)_{j} \mathrm{~d} s,
\end{array}
$$

where $\mathrm{d} s$ is the surface element of $\partial \Omega$. It turns out (see [11]) that $\Upsilon^{1}=\left(\Theta^{2}\right)^{\mathrm{T}}$ and that

$$
A=\left(\begin{array}{cc}
\bar{m}^{-1} \Theta^{1} & \bar{m}^{-1} \Theta^{2} \\
J^{-1} \Upsilon^{1} & J^{-1} \Upsilon^{2}
\end{array}\right)
$$

where $\bar{m}$ denotes the mass of the organism. Moreover, with respect to the inner product in $\mathbf{R}^{6}$ defined by

$$
\langle a, b\rangle_{J}=\bar{m} \sum_{l=1}^{3} a_{l} b_{l}+\sum_{l, k=1}^{3} J_{l k} a_{3+l} b_{3+k},
$$

$A$ is self-adjoint and negative-definite.

The matrix $B$ is defined by

where the entries of $\kappa$ and $\mathcal{K}$ are

$$
B=\left(\begin{array}{c}
\bar{m}^{-1} \kappa \\
J^{-1} \mathcal{K}
\end{array}\right)
$$

$$
\kappa_{i j}=-\int_{\partial \Omega} g^{(i)} \cdot \psi_{j} \mathrm{~d} s, \quad \mathcal{K}_{i j}=-\int_{\partial \Omega} G^{(i)} \cdot \psi_{j} \mathrm{~d} s,
$$

and $\psi_{1}, \ldots, \psi_{m}$ are fixed functions in $\mathcal{C}^{2}\left(\partial \Omega, \mathbf{R}^{3}\right)$, each one associated to a component of the control $u$, that describe the admissible propulsive actions of the organism.

\section{A Controllability Result in A more General SETting}

The following proposition provides sufficient (and necessary) conditions for the controllability of systems that generalize those introduced in the previous section, in the sense that a more general structure for the dynamics in the coordinates $z$ is allowed.

Proposition 4.1. Consider the following control system

$$
\dot{z}=f(z, u), \quad z \in \mathbf{R}^{6}, \quad u \in \mathbf{R}^{m} .
$$

Then (3.2), (3.3), (4.1) is controllable if and only if it is Lie-bracket generating at $(z, \zeta, R)=\left(0_{9}\right.$, Id) and system (4.1) is controllable. 
Proof. One direction of the equivalence is obvious, since an analytic controllable system is Lie bracket generating (see $[1,12])$.

Assume now that system (4.1) is controllable and that $(3.2),(3.3),(4.1)$ is Lie-bracket generating at $(z, \zeta, R)=$ $\left(0_{9}, \mathrm{Id}\right)$. Notice that

$$
\left(z^{\prime}, \zeta^{\prime}, R^{\prime}\right) \in \mathcal{A}(z, \zeta, R) \quad \Longrightarrow \quad\left(z^{\prime}, \bar{\zeta}+\bar{R} \zeta^{\prime}, \bar{R} R^{\prime}\right) \in \mathcal{A}(z, \bar{\zeta}+\bar{R} \zeta, \bar{R} R)
$$

for every $(\bar{\zeta}, \bar{R}) \in \mathbf{R}^{3} \times \mathrm{SO}(3)$, where the letter $\mathcal{A}$ is used here to denote attainable sets for system (3.2), (3.3), (4.1). Indeed, if $t \mapsto u(t)$ is an admissible control steering $(z, \zeta, R)$ to $\left(z^{\prime}, \zeta^{\prime}, R^{\prime}\right)$ and $t \mapsto(z(t), \zeta(t), R(t))$ is the corresponding trajectory, then $t \mapsto(z(t), \bar{\zeta}+\bar{R} \zeta(t), \bar{R} R(t))$ is an admissible trajectory corresponding to the same control $u$.

Fix $\left(z_{0}, \zeta_{0}, R_{0}\right),\left(z_{1}, \zeta_{1}, R_{1}\right) \in \mathbf{R}^{9} \times \mathrm{SO}(3)$. Since (4.1) is controllable, then there exist $\eta_{0}, \eta_{1} \in \mathbf{R}^{3}$ and $Q_{0}, Q_{1} \in \mathrm{SO}(3)$ such that $\left(0_{6}, \eta_{0}, Q_{0}\right) \in \mathcal{A}\left(z_{0}, \zeta_{0}, R_{0}\right)$ and $\left(z_{1}, \zeta_{1}, R_{1}\right) \in \mathcal{A}\left(0_{6}, \eta_{1}, Q_{1}\right)$. We are left to prove that $\mathcal{A}\left(0_{6}, \eta_{0}, Q_{0}\right)$ contains $\left\{0_{6}\right\} \times \mathbf{R}^{3} \times \mathrm{SO}(3)$.

Since $(3.2),(3.3),(4.1)$ is Lie-bracket generating at $(z, \zeta, R)=\left(0_{9}, \mathrm{Id}\right)$, then the set

$$
G=\operatorname{Int}\left(\mathcal{A}\left(0_{9}, \mathrm{Id}\right)\right)
$$

is nonempty, as it follows from Krener's theorem (see, for instance, [12], Thm. 1, p. 66). Fix $\left(z_{*}, \zeta_{*}, R_{*}\right) \in G$. Since (4.1) is controllable, there exists a control law $t \mapsto u(t)$, defined on an interval $[0, T]$ and such that the solution of (4.1) with initial condition $z(0)=z_{*}$ satisfies $z(T)=0_{6}$. The flow corresponding to the control law $u$, evaluated at time $T$, is a diffeomorphism sending $\left(z_{*}, \zeta_{*}, R_{*}\right)$ to a point of the form $\left(0_{6}, \zeta_{* *}, R_{* *}\right)$. In particular, since the image of $G$ by such diffeomorphism is contained in $G$, there exists an open nonempty subset $V$ of $\mathbf{R}^{3} \times \mathrm{SO}(3)$ such that $\left\{0_{6}\right\} \times V$ is contained in $G$.

Notice the following consequence of $(4.2)$ : if $\left(0_{6}, \bar{\zeta}, \bar{R}\right) \in \mathcal{A}\left(0_{6}, \eta_{0}, Q_{0}\right)$ and $\left(0_{6}, \zeta^{\prime}, R^{\prime}\right) \in G$, then

$$
\left(0_{6}, \bar{\zeta}+\bar{R} \zeta^{\prime}, \bar{R} R^{\prime}\right) \in \operatorname{Int}\left(\mathcal{A}\left(0_{6}, \eta_{0}, Q_{0}\right)\right) .
$$

Indeed, taking $(z, \zeta, R)=\left(0_{9}, \mathrm{Id}\right)$ in $(4.2)$, we have that for every $\left(z^{\prime \prime}, \zeta^{\prime \prime}, R^{\prime \prime}\right)$ in a neighborhood of $\left(0_{6}, \zeta^{\prime}, R^{\prime}\right)$, the point $\left(z^{\prime \prime}, \bar{\zeta}+\bar{R} \zeta^{\prime \prime}, \bar{R} R^{\prime \prime}\right)$ belongs to $\mathcal{A}\left(0_{6}, \bar{\zeta}, \bar{R}\right)$ and, since $\left(0_{6}, \bar{\zeta}, \bar{R}\right) \in \mathcal{A}\left(0_{6}, \eta_{0}, Q_{0}\right)$, we are done.

Notice that for every nonempty open subset $O$ of $\mathrm{SO}(3)$

$$
W=\left\{P_{1} P_{2} \ldots P_{k} \mid k \in \mathbf{N}, P_{1}, \ldots, P_{k} \in O\right\}
$$

is equal to $\mathrm{SO}(3)$. Indeed, since $O$ contains at least one element $P_{0}$ of finite order, i.e., an axial rotation of angle commensurable with $\pi$, then Id belongs to the interior of $W$. The completeness of $\operatorname{SO}(3)$ guarantees that $W=\mathrm{SO}(3)$.

Take as $O$ the projection of $V$ on $\mathrm{SO}(3)$. Then for every $R \in \mathrm{SO}(3)$ there exists $\zeta \in \mathbf{R}^{3}$ such that $\left(0_{6}, \zeta, R\right)$ belongs to $G$; this can be seen by noticing that there exist $k$ elements $\left(0_{6}, \zeta_{1}, P_{1}\right), \ldots,\left(0_{6}, \zeta_{k}, P_{k}\right)$ in $V$ such that $P_{i} \in O$ and $P_{1} P_{2} \ldots P_{k}=R$ and by applying repeatedly (4.3) in the special case $\eta_{0}=0_{3}$ and $Q_{0}=$ Id.

As a consequence, without loss of generality,

$$
Q_{0}=\mathrm{Id}, \quad Q_{1}=\mathrm{Id}
$$

Indeed, since there exists $\zeta_{0} \in \mathbf{R}^{3}$ such that $\left(0_{6}, \zeta_{0}, Q_{0}^{-1}\right) \in G$, equation (4.3) with $(\bar{\zeta}, \bar{R})=\left(\eta_{0}, Q_{0}\right)$ shows that $\left(0_{6}, \eta_{0}+Q_{0} \zeta_{0}, \mathrm{Id}\right) \in \mathcal{A}\left(0_{6}, \eta_{0}, Q_{0}\right)$; similarly, the existence of $\zeta_{1} \in \mathbf{R}^{3}$ such that $\left(0_{6}, \zeta_{1}, Q_{1}\right) \in G$ implies that $\left(0_{6}, \eta_{1}, Q_{1}\right) \in \mathcal{A}\left(0_{6}, \eta_{1}-\zeta_{1}, \mathrm{Id}\right)$.

Moreover, for every element $p$ of $\mathbf{R P}^{2}$, the Grassmannian of one-dimensional subspaces of $\mathbf{R}^{3}$, there exists $\zeta_{p} \in \mathbf{R}^{3}$ such that $\left(0_{6}, \zeta_{p}, R_{p}\right)$ lies in $G$, where $R_{p}$ denotes the rotation of angle $\pi$ around the axis $p$. Applying (4.3) to $(\bar{\zeta}, \bar{R})=\left(\zeta^{\prime}, R^{\prime}\right)=\left(\zeta_{p}, R_{p}\right)$ in the special case $\eta_{0}=0_{3}$, we obtain that $\left(0_{6}, \zeta_{p}+R_{p} \zeta_{p}\right.$, Id) lies in $G$. Notice that $\zeta_{p}+R_{p} \zeta_{p}=2\left\langle\zeta_{p}, p\right\rangle p$ belongs to the axis $p$. Therefore, $G$ intersects $\left\{0_{6}\right\} \times p \times\{\operatorname{Id}\}$ for every $p \in \mathbf{R P}^{2}$. 
Of special interest will be for us the expression of (4.3) when $\bar{R}=R^{\prime}=\mathrm{Id}$, namely

$$
\left(0_{6}, \bar{\zeta}, \mathrm{Id}\right) \in \mathcal{A}\left(0_{6}, \eta_{0}, \mathrm{Id}\right),\left(0_{6}, \zeta^{\prime}, \mathrm{Id}\right) \in G \quad \Longrightarrow \quad\left(0_{6}, \bar{\zeta}+\zeta^{\prime}, \mathrm{Id}\right) \in \operatorname{Int}\left(\mathcal{A}\left(0_{6}, \eta_{0}, \mathrm{Id}\right)\right)
$$

Let

$$
\mathcal{S}_{ \pm}^{2}=\left\{v \in \mathcal{S}^{2} \mid\left\{0_{6}\right\} \times \mathbf{R}_{\geq 0}( \pm v) \times\{\operatorname{Id}\} \cap G \neq \emptyset\right\}
$$

where $\mathcal{S}^{2}$ denotes the unit sphere in $\mathbf{R}^{3}$. As we remarked above, $\mathcal{S}_{+}^{2}$ and $\mathcal{S}_{-}^{2}$ cover $\mathcal{S}^{2}$ and, by construction, they are open. Moreover, both are nonempty, since $v \in \mathcal{S}_{ \pm}^{2}$ implies that $-v \in \mathcal{S}_{\mp}^{2}$. Since $\mathcal{S}^{2}$ is connected we have $\mathcal{S}_{+}^{2} \cap \mathcal{S}_{-}^{2} \neq \emptyset$, i.e., there exist $v \in \mathbf{R}^{3}$ and two non-negative constants $\lambda_{1}, \lambda_{2}$ such that both $\left(0_{6}, \lambda_{1} v\right.$, Id $)$ and $\left(0_{6},-\lambda_{2} v\right.$, Id) lie in $G$; moreover, due to the openness of $G$, these constants can be assumed to be positive and commensurable and so there exist two positive integers $n_{1}$ and $n_{2}$ such that $n_{1} \lambda_{1}-n_{2} \lambda_{2}=0$. Applying repeatedly (4.5) in the special case $\eta_{0}=0_{3}$, we obtain that $G$ contains $\left(0_{9}\right.$, Id) and, therefore, $\left\{0_{6}\right\} \times \mathbf{R}^{3} \times\{\operatorname{Id}\}$.

Applying (4.5) again with $\bar{\zeta}=\eta_{0}$ we deduce that $\left(0_{6}, \eta_{1}\right.$, Id $)$ is attainable from $\left(0_{6}, \eta_{0}\right.$, Id).

\section{Generic properties}

The expression generic is commonly used to denote a property of a system that is, in a suitable sense, stable under small perturbations and that, even when it fails to apply, can be forced to hold by applying to the system an arbitrary small perturbation.

In order to define precisely what a generic property means in the present context, define

$$
\Xi_{0}^{m}=\left\{(A, B, J) \in \mathscr{M}_{6 \times 6} \times \mathscr{M}_{6 \times m} \times \mathscr{M}_{3 \times 3} \mid J=J^{\mathrm{T}}>0, A \in \operatorname{Sym}_{J}^{-}\right\}
$$

where

$$
\operatorname{Sym}_{J}^{-}=\left\{A \in \mathscr{M}_{6 \times 6} \mid A \text { is symmetric and negative definite with respect to }\langle\cdot, \cdot\rangle_{J}\right\}
$$

and $\langle\cdot, \cdot\rangle_{J}$ is defined as in $(3.8)$.

We say that a property is generic for system (3.1)-(3.3) when $m=m_{0}$ if there exists an open and dense set $O$ in $\Xi_{0}^{m_{0}}$ such that the property holds for every system defined by a triple in $O$.

Such a definition of genericity, given in terms of the entries of the matrices $A, J$ and $B$, is adapted to the finite-dimensional formulation (3.1)-(3.3) but can appear unsatisfactory from the point of view of the physical model. Section 5.3 discusses how to define the genericity directly in the space of configurations of the organism and provides a physically justified counterpart of the following result.

Theorem 5.1. (a) Generically when $m \geq 1$ the rank of $\mathscr{L}$ is maximal at every point.

(b) Generically when $m \geq 3$ system (3.1)-(3.3) is controllable.

The generic conditions ensuring the Lie-bracket generating condition and the controllability of (3.1)-(3.3) are given explicitly in Propositions 5.2 and 5.3. Notice that the techniques applied in this section are independent of the structure imposed on $A$ by the physical motivations of the model, namely, the fact that $A \in \mathrm{Sym}_{J}^{-}$. Indeed, the conditions that are obtained in the following sections define an open and dense set in

$$
\Xi_{1}^{m}=\left\{(A, B, J) \in \mathscr{M}_{6 \times 6} \times \mathscr{M}_{6 \times m} \times \mathscr{M}_{3 \times 3} \mid J=J^{\mathrm{T}}>0\right\}
$$

for $m \geq 1$ (for the Lie-bracket generating condition) or $m \geq 3$ (for the controllability) whose intersection with $\Xi_{0}^{m}$ is open and dense in $\Xi_{0}^{m}$. In other words, Lie-bracket generating condition and controllability are generic properties (for $m=1$ and $m=3$, respectively) also in the class of systems of the form (3.1)-(3.3) for which the triple $(A, B, J)$ is taken in $\Xi_{1}^{m}$. 


\subsection{Generic Lie-bracket generating condition for $m=1$}

When $m=1$ the two matrices $B_{1}$ and $B_{2}$ are column vectors that we denote by $b_{1}$ and $b_{2}$ respectively. Then $X_{1}=\left(b_{1}, b_{2}, 0_{6}\right)$ and $\mathscr{L}$ is the Lie algebra generated by $X_{0}$ and $X_{1}$.

Proposition 5.2. Let $i=0$ or $i=1$. There exists an open and dense set of triples $(A, B, J)$ in $\Xi_{i}^{1}$ such that system (3.1)-(3.3) is Lie-bracket generating. More precisely, if $J, b_{1}$, and $b_{2}$ satisfy the three following conditions

$$
\begin{aligned}
\operatorname{det}\left(b_{1}, J b_{2}, J^{2} b_{2}\right) & \neq 0, \\
\operatorname{det}\left(b_{2}, J b_{2}, J^{2} b_{2}\right) & \neq 0, \\
\operatorname{det}\left(J-\frac{\left\|J b_{2}\right\|^{2}}{\left\langle J b_{2}, b_{2}\right\rangle} \text { Id }\right) & \neq 0,
\end{aligned}
$$

then $\mathscr{L}$ is maximal at every point.

Proof. Let us prove, first of all, that if $C \in \mathscr{M}(3 \times 3)$ is symmetric and invertible, and if $b \in \mathbf{R}^{3}$ is such that $C b$ and $C^{2} b$ are linearly independent, then

$$
C^{-1}(C b \times b)=\operatorname{det}\left(C^{-1}\right)\left(C^{2} b \times C b\right) .
$$

Notice that $\left\langle C^{-1}(C b \times b), C b\right\rangle=0=\left\langle C^{-1}(C b \times b), C^{2} b\right\rangle$, and therefore

$$
C^{-1}(C b \times b)=k\left(C^{2} b \times C b\right),
$$

for some $k \in \mathbf{R}$. For every $x \in \mathbf{R}^{3}$ we have $\operatorname{det}\left(C^{-1} x, C b, b\right)=\left(\operatorname{det} C^{-1}\right) \operatorname{det}\left(x, C^{2} b, C b\right)$ and also

$$
\operatorname{det}\left(C^{-1} x, C b, b\right)=\left\langle C^{-1} x, C b \times b\right\rangle=\left\langle x, C^{-1}(C b \times b)\right\rangle=k\left\langle x, C^{2} b \times C b\right\rangle=k \operatorname{det}\left(x, C^{2} b, C b\right) .
$$

Therefore

$$
\left(\operatorname{det} C^{-1}\right) \operatorname{det}\left(x, C^{2} b, C b\right)=k \operatorname{det}\left(x, C^{2} b, C b\right)
$$

for every $x \in \mathbf{R}^{3}$, proving that (5.4) holds true. In particular, taking $J=C$ and $b=b_{2}$, we have

$$
J^{-1}\left(J b_{2} \times b_{2}\right)=\operatorname{det}\left(J^{-1}\right)\left(J^{2} b_{2} \times J b_{2}\right) .
$$

Denote by $V_{1}$ the Lie bracket $\left[\left[X_{0}, X_{1}\right], X_{1}\right]$. According to (3.4) we have

$$
V_{1}=2\left(\left(b_{2} \times b_{1}\right), J^{-1}\left(J b_{2} \times b_{2}\right), 0_{6}\right) .
$$

Similarly, the definition $V_{2}=\frac{1}{4}\left[\left[X_{0}, V_{1}\right], V_{1}\right]$ leads to the expression

$$
V_{2}=\left(-\left\langle J^{-1}\left(J b_{2} \times b_{2}\right), b_{2}\right\rangle b_{1}+\left\langle J^{-1}\left(J b_{2} \times b_{2}\right), b_{1}\right\rangle b_{2},-\left\langle J^{-1}\left(J b_{2} \times b_{2}\right), b_{2}\right\rangle b_{2}, 0_{6}\right) .
$$

Since $\left\langle J^{-1}\left(J b_{2} \times b_{2}\right), b_{2}\right\rangle X_{1}+V_{2}=\left(\left\langle J^{-1}\left(J b_{2} \times b_{2}\right), b_{1}\right\rangle b_{2}, 0_{9}\right)$ we have that

$$
Z_{1}=\left(b_{2}, 0_{9}\right)
$$

belongs to $\mathscr{L}$, as it follows from (5.1) and from (5.6).

Let $Z_{2}=\frac{1}{2}\left[\left[X_{0}, Z_{1}\right], V_{1}\right]$. Applying again (3.4), we have that

$$
Z_{2}=\left(-b_{2} \times J^{-1}\left(J b_{2} \times b_{2}\right), 0_{9}\right) .
$$


Equation (5.6) implies that

$$
Z_{2}=\left(\left(\operatorname{det} J^{-1}\right) b_{2} \times\left(J b_{2} \times J^{2} b_{2}\right), 0_{9}\right)
$$

Letting

$$
b_{3}=b_{2} \times\left(J b_{2} \times J^{2} b_{2}\right)
$$

we can represent $Z_{3}=\frac{1}{2}\left[\left[X_{0}, Z_{2}\right], X_{1}\right]$ as

$$
Z_{3}=\left(b_{2} \times b_{3}, 0_{9}\right) .
$$

We have shown that the Lie algebra $\mathscr{L}$ contains the vector fields

$$
Z_{1}=\left(b_{2}, 0_{9}\right), \quad Z_{2}=\left(b_{3}, 0_{9}\right), \quad Z_{3}=\left(b_{2} \times b_{3}, 0_{9}\right) .
$$

Notice that

$$
b_{3}=\left\langle b_{2}, J^{2} b_{2}\right\rangle J b_{2}-\left\langle b_{2}, J b_{2}\right\rangle J^{2} b_{2}
$$

is not in $\operatorname{span}\left(b_{2}\right)$, since $\left\langle b_{2}, J b_{2}\right\rangle>0$ and because of (5.2). As a consequence, the vectors $b_{2}, b_{3}$ and $b_{2} \times b_{3}$ are linearly independent.

Therefore, $\mathscr{L}$ contains every constant vector field of the type $\left(v, 0_{9}\right)$, with $v$ in $\mathbf{R}^{3}$. It follows that

$$
W_{1}=\left(0_{3}, b_{2}, 0_{6}\right)
$$

is in $\mathscr{L}$. As a consequence of $(3.4)$, [[ $\left.\left.X_{0}, W_{1}\right], W_{1}\right]=2\left(0_{3}, J^{-1}\left(J b_{2} \times b_{2}\right), 0_{6}\right)$ lies in $\mathscr{L}$ as well. Due to (5.6), we deduce that

$$
W_{2}=\left(0_{3}, J^{2} b_{2} \times J b_{2}, 0_{6}\right)
$$

belongs to $\mathscr{L}$. Let

$$
b_{4}=J^{2} b_{2} \times J b_{2}
$$

and let $W_{3}=\left[\left[X_{0}, W_{1}\right], W_{2}\right]$; we have $W_{3}=\left(0_{3}, b_{5}, 0_{6}\right)$ with $b_{5}=J^{-1}\left(J b_{4} \times b_{2}+J b_{2} \times b_{4}\right)$. We are left to prove that the vectors $b_{2}, b_{4}$ and $b_{5}$ are linearly independent. Let $D=\operatorname{det}\left(b_{2}, b_{4}, b_{5}\right)$ and notice that

$$
\begin{aligned}
D & =\operatorname{det}\left(b_{2}, b_{4}, J^{-1}\left(J b_{4} \times b_{2}+J b_{2} \times b_{4}\right)\right) \\
& =\left(\operatorname{det} J^{-1}\right) \operatorname{det}\left(J b_{2}, J b_{4}, J b_{4} \times b_{2}+J b_{2} \times b_{4}\right) \\
& =\left(\operatorname{det} J^{-1}\right)\left\langle J b_{2}, J b_{4} \times\left(J b_{4} \times b_{2}\right)+J b_{4} \times\left(J b_{2} \times b_{4}\right)\right\rangle \\
& =\left(\operatorname{det} J^{-1}\right)\left\langle J b_{2},\left\langle J b_{4}, b_{4}\right\rangle J b_{2}-\left\langle J b_{4}, J b_{2}\right\rangle b_{4}+\left\langle J b_{4}, b_{2}\right\rangle J b_{4}-\left\|J b_{4}\right\|^{2} b_{2}\right\rangle .
\end{aligned}
$$

Since $\left\langle J b_{4}, J b_{2}\right\rangle=\left\langle b_{4}, J^{2} b_{2}\right\rangle=0$ and $\left\langle J b_{4}, b_{2}\right\rangle=\left\langle b_{4}, J b_{2}\right\rangle=0$ we have

$$
\begin{aligned}
D & =\left(\operatorname{det} J^{-1}\right)\left\langle J b_{2},\left\langle J b_{4}, b_{4}\right\rangle J b_{2}-\left\|J b_{4}\right\|^{2} b_{2}\right\rangle \\
& =\left(\operatorname{det} J^{-1}\right)\left(\left\|J b_{2}\right\|^{2}\left\langle J b_{4}, b_{4}\right\rangle-\left\|J b_{4}\right\|^{2}\left\langle J b_{2}, b_{2}\right\rangle\right) .
\end{aligned}
$$

Now, taking $C=J^{-1}$ and $b=J^{2} b_{2}$ in (5.4), we have $J b_{4}=(\operatorname{det} J)\left(J b_{2} \times b_{2}\right)$, from which we deduce

$$
\left\langle J b_{4}, b_{4}\right\rangle=(\operatorname{det} J)\left(\left\langle J b_{2}, b_{2}\right\rangle\left\langle J b_{2}, J^{2} b_{2}\right\rangle-\left\|J b_{2}\right\|^{4}\right) .
$$

We also have

$$
\begin{aligned}
\left\|J b_{4}\right\|^{2} & =(\operatorname{det} J)^{2}\left\|J b_{2} \times b_{2}\right\|^{2} \\
& =(\operatorname{det} J)^{2}\left(\left\|J b_{2}\right\|^{2}\left\|b_{2}\right\|^{2}-\left\langle J b_{2}, b_{2}\right\rangle^{2}\right) .
\end{aligned}
$$


Defining $\lambda=\left\|J b_{2}\right\|^{2} /\left\langle J b_{2}, b_{2}\right\rangle$ we get

$$
\begin{aligned}
D & =\left\|J b_{2}\right\|^{2}\left(\left\langle J b_{2}, b_{2}\right\rangle\left\langle J^{2} b_{2}, J b_{2}\right\rangle-\left\|J b_{2}\right\|^{4}\right)-(\operatorname{det} J)\left\langle J b_{2}, b_{2}\right\rangle\left(\left\|J b_{2}\right\|^{2}\left\|b_{2}\right\|^{2}-\left\langle J b_{2}, b_{2}\right\rangle^{2}\right) \\
& =\left\langle J b_{2}, b_{2}\right\rangle^{3}\left(\frac{\left\langle J^{3} b_{2}, b_{2}\right\rangle}{\left\langle J b_{2}, b_{2}\right\rangle} \lambda-\lambda^{3}-(\operatorname{det} J)\left(\frac{\left\|b_{2}\right\|^{2}}{\left\langle J b_{2}, b_{2}\right\rangle} \lambda-1\right)\right) .
\end{aligned}
$$

Cayley-Hamilton theorem implies that

$$
J^{3}=a_{2} J^{2}+a_{1} J+(\operatorname{det} J) \mathrm{Id}, \quad a_{1}, a_{2} \in \mathbf{R},
$$

and thus

$$
\begin{aligned}
\frac{\left\langle J^{2} b_{2}, J b_{2}\right\rangle}{\left\langle J b_{2}, b_{2}\right\rangle} & =a_{2} \frac{\left\langle J^{2} b_{2}, b_{2}\right\rangle}{\left\langle J b_{2}, b_{2}\right\rangle}+a_{1}+(\operatorname{det} J) \frac{\left\|b_{2}\right\|^{2}}{\left\langle J b_{2}, b_{2}\right\rangle} \\
& =a_{2} \lambda+a_{1}+(\operatorname{det} J) \frac{\left\|b_{2}\right\|^{2}}{\left\langle J b_{2}, b_{2}\right\rangle} .
\end{aligned}
$$

Therefore,

$$
\begin{aligned}
D & =\left\langle J b_{2}, b_{2}\right\rangle^{3}\left(\left(a_{2} \lambda+a_{1}+(\operatorname{det} J) \frac{\left\|b_{2}\right\|^{2}}{\left\langle J b_{2}, b_{2}\right\rangle}\right) \lambda-\lambda^{3}-(\operatorname{det} J)\left(\frac{\left\|b_{2}\right\|^{2}}{\left\langle J b_{2}, b_{2}\right\rangle} \lambda-1\right)\right) \\
& =\left\langle J b_{2}, b_{2}\right\rangle^{3}\left(-\lambda^{3}+a_{2} \lambda^{2}+a_{1} \lambda+\operatorname{det} J\right) \\
& =\left\langle J b_{2}, b_{2}\right\rangle^{3} \operatorname{det}(J-\lambda \mathrm{Id})
\end{aligned}
$$

which is different from zero because of (5.3).

\subsection{Generic controllability in the case $m=3$}

The aim of this section is to prove the genericity of the controllability of (3.1) in the case $m=3$. Let us introduce the notation

with each $A_{i j}$ belonging to $\mathscr{M}_{3 \times 3}$.

$$
A=\left(\begin{array}{ll}
A_{11} & A_{12} \\
A_{21} & A_{22}
\end{array}\right)
$$

Proposition 5.3. Let $i=0$ or $i=1$. There exists an open and dense set of triples $(A, B, J)$ in $\Xi_{i}^{3}$ such that system (3.1) is controllable. More precisely, if $B_{2}$ and $\tilde{A}_{11}+\tilde{A}_{11}^{\mathrm{T}}$ are invertible with $\tilde{A}_{11}=A_{11}-B_{1} B_{2}^{-1} A_{21}$ and if at least one eigenvector of $J$ is not an eigenvector of $B_{1} B_{2}^{-1}$, then system (3.1) is controllable.

Propositions 5.2 and 5.3 lead to the following statement, which follows from Proposition 4.1.

Corollary 5.4. For $m \geq 3$ and $i=0$ or $i=1$, there exists an open and dense set of triples $(A, B, J)$ in $\Xi_{i}^{3}$ such that system (3.1)-(3.3) is controllable.

The proof of Proposition 5.3 will be split in several steps. The following lemma allows us to study the controllability of (3.1) by investigating the controllability of an equivalent system in $\mathbf{R}^{3}$ (instead of $\mathbf{R}^{6}$ ).

Lemma 5.5. Assume that $B_{2}$ is invertible and define $\tilde{B}=B_{1} B_{2}^{-1}$ and $\tilde{A}_{11}=A_{11}-\tilde{B} A_{21}$. Then, the controllability of (3.1) is equivalent to the controllability of the system

$$
\dot{x}=\tilde{A}_{11} x-v \times x+\left(\tilde{A}_{11} \tilde{B}+A_{12}-\tilde{B} A_{22}\right) v-v \times \tilde{B} v-\tilde{B} J^{-1}(J v \times v), \quad x \in \mathbf{R}^{3},
$$

with the control $v$ taking values in $\mathbf{R}^{3}$. 
Proof. Suppose that system (3.1) is controllable. Let $x=\xi-\tilde{B} \omega$ and rewrite system (3.1) as

$$
\left\{\begin{array}{l}
\dot{x}=\tilde{A}_{11} x-\omega \times x+\left(\tilde{A}_{11} \tilde{B}+A_{12}-\tilde{B} A_{22}\right) \omega-\omega \times \tilde{B} \omega-\tilde{B} J^{-1}(J \omega \times \omega), \\
\dot{\omega}=A_{21} x+\left(A_{21} \tilde{B}+A_{22}\right) \omega+J^{-1}(J \omega \times \omega)+B_{2} u .
\end{array}\right.
$$

Given two points $\left(x_{0}, \omega_{0}\right)$ and $\left(x_{1}, \omega_{1}\right)$ in $\mathbf{R}^{6}$, there exists a control $u:[0, T] \rightarrow \mathbf{R}^{3}$ that steers $\left(x_{0}, \omega_{0}\right)$ to $\left(x_{1}, \omega_{1}\right)$. Thus, denoting by $(x(\cdot), \omega(\cdot))$ the corresponding trajectory, the control $v(t)=\omega(t)$ steers system (5.7) from $x_{0}$ to $x_{1}$.

Conversely, let system (5.7) be controllable and fix two pairs $\left(\xi_{0}, \omega_{0}\right)$ and $\left(\xi_{1}, \omega_{1}\right)$ in $\mathbf{R}^{6}$. Notice that (5.7) can be controlled by smooth controls $v:[0, T] \rightarrow \mathbf{R}^{3}$ satisfying

$$
v(0)=\omega_{0}, \quad v(T)=\omega_{1}
$$

(The result follows from the controllability of (5.7) and the density of $\left\{v \in \mathcal{C}^{\infty}\left([0, T], \mathbf{R}^{3}\right) \mid v(0)=\omega_{0}\right.$, $\left.v(T)=\omega_{1}\right\}$ in $L^{\infty}(0, T)$ with respect to the $L^{1}$-norm. The proof can be deduced from the general results in [10] or easily adapted from [6] and [12], Thm. 4, p. 110.)

Therefore, if $v \in \mathcal{C}^{\infty}\left([0, T], \mathbf{R}^{3}\right)$ satisfies (5.8) and steers $(5.7)$ from $\xi_{0}-\tilde{B} \omega_{0}$ to $\xi_{1}-\tilde{B} \omega_{1}$, then

$$
u(t)=B_{2}^{-1}\left(\dot{v}(t)-A_{21} x(t)-\left(A_{21} \tilde{B}+A_{22}\right) v(t)-J^{-1}(J v(t) \times v(t))\right)
$$

steers $(3.1)$ from $\left(\xi_{0}, \omega_{0}\right)$ to $\left(\xi_{1}, \omega_{1}\right)$.

The following lemma establishes the controllability of system (5.7) in a first case.

Lemma 5.6. Assume that the matrix $\tilde{A}_{11}+\tilde{A}_{11}^{\mathrm{T}}$ has two nonzero eigenvalues with opposite signs. Then there exists an open and dense subset $O$ of $\left\{(B, J) \in \mathscr{M}_{6 \times 3} \times \mathscr{M}_{3 \times 3} \mid J=J^{\mathrm{T}}>0\right\}$ such that system (5.7) is controllable if $(B, J) \in O$. More precisely, if $B_{2}$ is invertible and if at least one eigenvector of $J$ is not an eigenvector of $B_{1} B_{2}^{-1}$, then system (5.7) is controllable.

For the proof of this lemma we shall use the following result, proven in [15], about the controllability of a family of affine vector fields in $\mathbf{R}^{n}$. An affine vector field $X$ is a mapping from $\mathbf{R}^{n}$ to $\mathbf{R}^{n}$ of the form $X: x \mapsto C x+c$ where $C \in \mathscr{M}_{n \times n}$ and $c$ is a constant vector in $\mathbf{R}^{n}$; the linear part of $X$, denoted by $\Lambda(X)$, is the linear vector field $x \mapsto C x$. If $\mathscr{F}$ is a family of affine vector fields, we denote by $\Lambda(\mathscr{F})$ the set of linear parts of the vector fields in $\mathscr{F}$ and we say that $\mathscr{F}$ has no fixed point if there does not exist a point $x_{0} \in \mathbf{R}^{n}$ such that $X\left(x_{0}\right)=0$ for every $X \in \mathscr{F}$.

Theorem 5.7 (Jurdjevic and Sallet). Let $\mathscr{F}$ be a family of affine vector fields given on $\mathbf{R}^{n}$. Assume that $\mathscr{F}$ has no fixed point. If $\Lambda(\mathscr{F})$ is controllable on $\mathbf{R}^{n} \backslash\{0\}$ then $\mathscr{F}$ is controllable on $\mathbf{R}^{n}$.

Proof of Lemma 5.6. From Lemma 5.5, we know that it is sufficient to prove the controllability of the system defined by the family of affine vector fields $\mathscr{F}=\left\{C(v) x+c(v) \mid v \in \mathbf{R}^{3}\right\}$ where

$$
C(v)=\tilde{A}_{11}-S(v), \quad c(v)=\left(\tilde{A}_{11} \tilde{B}+A_{12}-\tilde{B} A_{22}\right) v-v \times \tilde{B} v-\tilde{B} J^{-1}(J v \times v) .
$$

Choose $v_{0}$ in such a way that $S\left(v_{0}\right)=\left(\tilde{A}_{11}-\tilde{A}_{11}^{\mathrm{T}}\right) / 2$. Then

$$
\tilde{A}_{11}-S\left(v_{0}\right)=\frac{\tilde{A}_{11}+\tilde{A}_{11}^{\mathrm{T}}}{2}
$$

Define the family of linear vector fields $\mathscr{G}=\left\{\tilde{A}_{11}-S\left(v_{0}\right), S(v) \mid v \in \mathbf{R}^{3}\right\}$. The two closed convex cones generated by the families $\Lambda(\mathscr{F})$ and $\mathscr{G}$ are identical, since for every finite family $\left(\alpha_{i}\right)_{0 \leq i \leq N}$ of positive numbers 
and every choice of $v_{1}, \ldots, v_{N} \in \mathbf{R}^{3}$,

$$
\alpha_{0}\left(\tilde{A}_{11}-S\left(v_{0}\right)\right)+\sum_{i=1}^{N} \alpha_{i} S\left(v_{i}\right)=\alpha_{0}\left(\tilde{A}_{11}-S\left(v_{0}\right)\right)+\lim _{r \rightarrow+\infty} \frac{1}{r} \sum_{i=1}^{N} \alpha_{i}\left(\tilde{A}_{11}-S\left(-r v_{i}\right)\right)
$$

and

$$
\sum_{i=1}^{N} \alpha_{i}\left(\tilde{A}_{11}-S\left(v_{i}\right)\right)=\left(\sum_{i=1}^{N} \alpha_{i}\right)\left(\tilde{A}_{11}-S\left(v_{0}\right)\right)+\sum_{i=1}^{N} \alpha_{i} S\left(v_{0}-v_{i}\right) .
$$

Therefore, the controllability of $\Lambda(\mathscr{F})$ on $\mathbf{R}^{3} \backslash\{0\}$ is equivalent to the one of $\mathscr{G}$ (see $[13,14]$ ).

The trajectories of the vector field $S(v)$ are circles contained in planes orthogonal to $v$ and whose centers are at the intersections of these planes with the line $\mathbf{R} v$. Let $x \in \mathbf{R}^{3} \backslash\{0\}$; thanks to the vector fields $S(v)$, the attainable set from $x$ for $\mathscr{G}$ contains the sphere of center $0_{3}$ passing through $x$. Now, as $\tilde{A}_{11}-S\left(v_{0}\right)$ has two eigenvalues with opposite sign, we can move (thanks to this vector field) along a direction towards the origin and along an half-line exiting the sphere and going to infinity. Therefore, using again the fact that the family $\left\{S(v) \mid v \in \mathbf{R}^{3}\right\}$ is transitive on every sphere, we proved that $\mathscr{G}$, and thus $\Lambda(\mathscr{F})$, is controllable on $\mathbf{R}^{3} \backslash\{0\}$.

Assume now that we can find a fixed point, denoted by $x_{0}$, which is common to all the vector fields in $\mathscr{F}$. For every vector $v \in \mathbf{R}^{3}$, we have

$$
C(v) x_{0}+c(v)=C(-v) x_{0}+c(-v)=0_{3}
$$

from which we deduce

$$
\tilde{A}_{11} x_{0}-v \times \tilde{B} v-\tilde{B} J^{-1}(J v \times v)=0 .
$$

Taking $v=0_{3}$ gives $\tilde{A}_{11} x_{0}=0_{3}$ and therefore (5.9) can be rewritten as

$$
\tilde{B} v \times v=\tilde{B} J^{-1}(J v \times v)
$$

for every $v \in \mathbf{R}^{3}$. In particular, if $v$ is an eigenvector of $J$ but not of $\tilde{B}$, then the right-hand side of (5.10) is equal to zero, while the right-hand side is not, leading to a contradiction.

We are left to deal with the case where the eigenvalues of

$$
\tilde{A}_{11}^{s}=\frac{\tilde{A}_{11}+\tilde{A}_{11}^{\mathrm{T}}}{2}
$$

the symmetric part of $\tilde{A}_{11}$, are all positive or all negative. Although in this case the linear part of the family $\mathscr{F}$ is not controllable on $\mathbf{R}^{3} \backslash\{0\}$, we can nevertheless adapt the method introduced in [15] in order to prove the controllability of (5.7).

Lemma 5.8. Assume that the matrix $\tilde{A}_{11}^{s}$ is invertible and that its eigenvalues have all the same sign. Then there exists an open and dense subset $O$ of $\left\{(B, J) \in \mathscr{M}_{6 \times 3} \times \mathscr{M}_{3 \times 3} \mid J=J^{\mathrm{T}}>0\right\}$ such that system (5.7) is controllable if $(B, J) \in O$. More precisely, if $B_{2}$ is invertible and if at least one eigenvector of $J$ is not an eigenvector of $\tilde{B}$, then system (5.7) is controllable.

Notice that, together with Lemma 5.6, Lemma 5.8 concludes the proof of Proposition 5.3. In order to show Lemma 5.8 we need some definitions and have to prove some intermediate results.

Let $\mathscr{F}$ and $\Lambda(\mathscr{F})$ be defined as above. In the proof of Lemma 5.6 we pointed out that starting form a point $x \in \mathbf{R}^{3}$ and following all possible linear vector fields of the type $S(v)$ one can attain the entire sphere of center $0_{3}$ 
and radius $\|x\|$. If the eigenvalues of $\tilde{A}_{11}^{s}$ are negative (resp. positive), the vector field $\tilde{A}_{11}^{s}$ points towards the interior (resp. the exterior) of this sphere, from which we deduce that

$$
\mathcal{A}(x, \Lambda(\mathscr{F}))= \begin{cases}\{x\} \cup\left\{y \in \mathbf{R}^{3} \mid 0<\|y\|<\|x\|\right\} & \text { if } \tilde{A}_{11}^{s}<0 \\ \{x\} \cup\left\{y \in \mathbf{R}^{3} \mid\|y\|>\|x\|\right\} & \text { if } \tilde{A}_{11}^{s}>0\end{cases}
$$

In the sequel, we shall need the notion of normal accessibility, which is recalled below.

Definition 5.9. Let $\mathscr{V}$ be a family of complete vector fields on a manifold $M$. The point $y$ is said to be normally $\mathscr{V}$-accessible from $x$ if there exist $X^{1}, \ldots, X^{p}$ in $\mathscr{V}$ and $t_{1}, \ldots, t_{p}>0$ such that

$$
y=\mathrm{e}^{t_{1} X^{1}} \circ \cdots \circ \mathrm{e}^{t_{p} X^{p}}(x)
$$

and the mapping

$$
\left(\tau_{1}, \ldots, \tau_{p}\right) \longmapsto \mathrm{e}^{\tau_{1} X^{1}} \circ \cdots \circ \mathrm{e}^{\tau_{p} X^{p}}(x),
$$

defined in a neighborhood of $\left(t_{1}, \ldots, t_{p}\right)$, is of rank equal to $\operatorname{dim} M$ at $\left(t_{1}, \ldots, t_{p}\right)$.

In [15] the authors exploit the fact that if a family of vector fields is controllable, then every point $y$ is normally accessible from every point $x$. As the family $\Lambda(\mathscr{F})$ is not controllable on $\mathbf{R}^{3} \backslash\{0\}$, we are lead to prove a suitable normal accessibility property. First, we recall the notion of projecting a linear vector field on the unit sphere. For every linear vector field $U$ defined on $\mathbf{R}^{n}$, the projection of $U$ on the unit sphere $S^{n-1}$ is the vector field $\bar{U}$ defined, for every $x \in S^{n-1}$, as

$$
\bar{U}(x)=U(x)-\langle U(x), x\rangle x .
$$

In the particular case where $n=3$ and $U(x)=S(\omega) x$, we have $\bar{U}=\left.U\right|_{S^{2}}$. Denote by $\bar{S}(\omega)$ the vector field obtained in this way. Notice that if $S(w)$ is nonzero, then its spectrum contains the value zero and two nonzero purely imaginary eigenvalues. So, the phase portrait of $\bar{S}(\omega)$ consists of all circles obtained as intersections of $S^{2}$ with the planes orthogonal to $\omega$ (and in particular the two singletons $\pm \omega /\|\omega\|$ ).

Going back to the general case, it can easily be seen that the flow of $\bar{U}$ is given by the expression $\frac{\mathrm{e}^{t U} x}{\left\|\mathrm{e}^{t U}\right\|}$. This means that the integral curves of $\bar{U}$ are obtained by projecting the integral curves of $U$ onto $S^{n-1}$. This leads to the following remark: let $\mathscr{H}$ be a family of linear vector fields on $\mathbf{R}^{n}$ and denote by $\overline{\mathscr{H}}$ the family of their projections on $S^{n-1}$. Then $\overline{\mathscr{H}}$ is controllable on $S^{n-1}$ if and only if $\mathscr{H}$ is transitive on directions, i.e., any ray from the origin is reachable by $\mathscr{H}$ from any other ray from the origin.

Lemma 5.10. Assume that the eigenvalues of $\tilde{A}_{11}+\tilde{A}_{11}^{\mathrm{T}}$ have all the same sign. Then we can extract from $\mathscr{F}$ a finite family $\mathscr{F}^{0}$ such that there exists a sphere of center $0_{3}$ and radius $r$ of points normally $\Lambda\left(\mathscr{F}^{0}\right)$-accessible from every point of the unit sphere.

Proof. Let $v_{0} \in \mathbf{R}^{3}$ be such that $\tilde{A}_{11}^{s}=\tilde{A}_{11}-S\left(v_{0}\right)=\tilde{A}_{11}^{s}$. Let, moreover, $w_{1}$ be an eigenvector of $\tilde{A}_{11}^{s}$ and take $w_{2}, w_{3} \in \mathbf{R}^{3}$ such that the family $\left(w_{1}, w_{2}, w_{3}\right)$ is a basis of $\mathbf{R}^{3}$. Consider now the family

$$
\Lambda\left(\mathscr{F}^{0}\right)=\left\{\tilde{A}_{11}^{s}, \tilde{A}_{11}^{s}-k_{2} S\left(w_{2}\right), \tilde{A}_{11}^{s}-k_{3} S\left(w_{3}\right)\right\}
$$

extracted from $\Lambda(\mathscr{F})$, with $k_{2}$ and $k_{3}$ to be chosen later.

Since $\omega_{2}$ and $\omega_{3}$ are linearly independent, then the family $\left\{\bar{S}\left(\omega_{2}\right), \bar{S}\left(\omega_{3}\right)\right\}$ is controllable on $S^{2}$. Notice now that

$$
\lim _{k \rightarrow+\infty} \frac{\tilde{A}_{11}^{s}-k S\left(w_{j}\right)}{k}=-S\left(w_{j}\right)
$$

and that the projection on $S^{2}$ of the linear vector field corresponding to $\tilde{A}_{11}^{s}-k S\left(w_{j}\right) / k$ converges to $-\bar{S}\left(w_{j}\right)$ as $k$ goes to infinity. Since the controllability is an open property (see [21]), we deduce that for $k_{2}$ and $k_{3}$ large 
enough the projection onto $S^{2}$ of the family $\left\{\left(\tilde{A}_{11}^{s}-k_{2} S\left(w_{2}\right)\right) / k_{2},\left(\tilde{A}_{11}^{s}-k_{3} S\left(w_{3}\right)\right) / k_{3}\right\}$ is controllable on $S^{2}$, which implies that the family $\left\{\left(A_{11}-k_{2} S\left(w_{2}\right)\right) / k_{2},\left(A_{11}-k_{3} S\left(w_{3}\right)\right) / k_{3}\right\}$ is transitive on directions. Since the integral curves of $\left(A_{11}-k_{j} S\left(w_{j}\right)\right) / k_{j}$ coincide with those of $A_{11}-k_{j} S\left(\omega_{j}\right)$ for $j=2,3$, we can conclude that the family $\left\{A_{11}-k_{2} S\left(w_{2}\right), A_{11}-k_{3} S\left(w_{3}\right)\right\}$ is transitive on directions, provided that $k_{2}$ and $k_{3}$ are chosen large enough.

Moreover, any nontrivial trajectory of the vector field $\tilde{A}_{11}^{s}$ whose initial condition belongs to its eigendirection $\operatorname{span}\left(w_{1}\right)$ either diverges to infinity (case $\tilde{A}_{11}^{s}>0$ ) or converges to the origin (case $\tilde{A}_{11}^{s}<0$ ). Hence, for every $x \in \mathbf{R}^{3} \backslash\left\{0_{3}\right\}, \mathcal{A}\left(x, \Lambda\left(\mathscr{F}^{0}\right)\right)$ contains a set of the form $\mathbf{R}^{3} \backslash B(0, r)$ (case $\tilde{A}_{11}^{s}>0$ ) or $B(0, r) \backslash\left\{0_{3}\right\}$ (case $\left.\tilde{A}_{11}^{s}<0\right)$. The interior of $\mathcal{A}\left(x, \Lambda\left(\mathscr{F}^{0}\right)\right)$ being nonempty, there exists at least one point, denoted by $y$, that can be normally $\Lambda\left(\mathscr{F}^{0}\right)$-accessed from $x$ (see [21]). Notice that since $y$ is normally $\Lambda\left(\mathscr{F}^{0}\right)$-accessible from $x$, then $y$ is also normally $\Lambda\left(\mathscr{F}^{0}\right)$-accessible from every point in a (sufficiently small) neighborhood of $x$. Moreover, all the points in $\mathcal{A}\left(y, \Lambda\left(\mathscr{F}^{0}\right)\right)$ are normally $\Lambda\left(\mathscr{F}^{0}\right)$-accessible from $x$.

We conclude by using the compactness of the unit sphere: for every $x \in \mathcal{S}^{2}$, there exists a neighborhood $V_{x}$ of $x$ and a set $\mathbf{R}^{3} \backslash B\left(0, r_{x}\right)$ (case $\left.\tilde{A}_{11}^{s}>0\right)$ or $B\left(0, r_{x}\right) \backslash\left\{0_{3}\right\}$ (case $\left.\tilde{A}_{11}^{s}<0\right)$ whose points are normally $\Lambda\left(\mathscr{F}^{0}\right)$ accessible from every point of $V_{x}$. As we can include the unit sphere in a finite union of neighborhoods $V_{x}$, we can claim the existence of a sphere of radius $r$ whose points are normally $\Lambda\left(\mathscr{F}^{0}\right)$-accessible from every point of the unit sphere.

The following lemma guarantees the unboundedness of the sets $\mathcal{A}(x, \mathscr{F})$ and $\mathcal{A}(x,-\mathscr{F})$.

Lemma 5.11. If $B_{2}$ is invertible and if at least one eigenvector of $J$ is not an eigenvector of $\tilde{B}$, then for every $x \in \mathbf{R}^{3}$ both sets $\mathcal{A}(x, \mathscr{F})$ and $\mathcal{A}(x,-\mathscr{F})$ are unbounded.

Proof. Take $v \in \mathbf{R}^{3}$ such that $q(v)=-v \times \tilde{B} v-\tilde{B} J^{-1}(J v \times v) \neq 0_{3}$; this is possible since at least one eigenvector of $J$ is not an eigenvector of $\tilde{B}$. For every $\alpha \in \mathbf{R}$ the vector field $x \mapsto C(\alpha v) x+c(\alpha v)$ is in $\mathscr{F}$ and

$$
\lim _{\alpha \rightarrow \infty} \frac{C(\alpha v) x+c(\alpha v)}{\alpha^{2}}=q(v) .
$$

This proves that, given a time $T>0$, the solution of $\dot{x}=C(\alpha v) x+c(\alpha v)$ on the interval $\left[-T \alpha^{-2}, T \alpha^{-2}\right]$ is as close as we want to the solution of $\dot{x}=q(v)$ on $[-T, T]$ (with the same initial condition) provided that $\alpha$ is large enough. Now, the solution of $\dot{x}=q(v), x(0)=x_{0}$, leaves any fixed bounded set both in time $T$ and in time $-T$, provided that $T$ is large enough.

We consider now, as in [15], the family $\left\{h_{\lambda, w} \mid \lambda>0, w \in \mathbf{R}^{n}\right\}$ of affine diffeomorphisms of $\mathbf{R}^{n}$ defined by $h_{\lambda, w}(x)=w+\lambda(x-w)$. An easy computation shows that, for every $X \in \mathscr{F}$,

$$
\left(h_{\lambda, w_{*}} X\right)(x)=\Lambda(X)(x-w)+\lambda X(w)
$$

where $h_{\lambda, w_{*}} X$ denotes the pushforward of the vector field $X$ by $h_{\lambda, w}$. In particular

$$
\lim _{\lambda \rightarrow 0}\left(h_{\lambda, w_{*}} X\right)(x)=\Lambda(X)(x-w) .
$$

Denote by $\mathscr{F}_{\lambda, w}$ the image of the family $\mathscr{F}$ by $h_{\lambda, w_{*}}$, that is, the transformation of $\mathscr{F}$ under the change of coordinates $h_{\lambda, w}$.

Proof of Lemma 5.8. Let $\Lambda\left(\mathscr{F}^{0}\right)$ and $r$ be as in the statement of Lemma 5.10. Consider the finite family $\Lambda\left(\mathscr{F}_{w}^{0}\right)=\left\{\Lambda(X)(x-w) \mid X \in \mathscr{F}^{0}\right\}$ and notice that each point of the sphere of center $w$ and radius $r$ is normally $\Lambda\left(\mathscr{F}_{w}^{0}\right)$-accessible from every point of the sphere of center $w$ and radius 1 . Let $\mathscr{F}_{\lambda, w}=\left\{h_{\lambda, w_{*}} X \mid X \in \mathscr{F}\right\}$. Thanks to formula (5.11) we can assert that, if $\lambda$ is chosen sufficiently small, the sphere of center $w$ and radius $r$ is contained in the attainable set for $\mathscr{F}_{\lambda, w}$ from every point of the sphere of center $w$ and radius 1 (see [21], Lem. 3.2). Fix such a $\lambda>0$. 


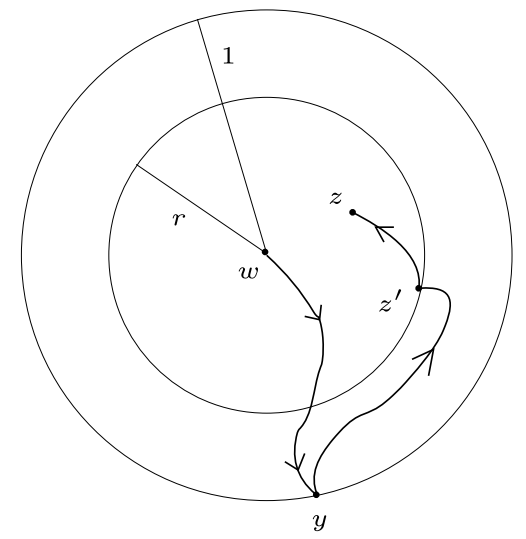

Figure 1. $z$ belongs to $\mathcal{A}\left(w, \mathscr{F}_{\lambda, w}\right)$ (the case $\left.r<1\right)$.

Given $w$ in $\mathbf{R}^{3}$ we claim that $\mathcal{A}(w, \mathscr{F})$ contains a neighborhood of $w$. Indeed, since the sets $\mathcal{A}(x, \mathscr{F})$ and $\mathcal{A}(x,-\mathscr{F})$ are unbounded, the same is true for the sets $\mathcal{A}\left(x, \mathscr{F}_{\lambda, w}\right)$ and $\mathcal{A}\left(x,-\mathscr{F}_{\lambda, w}\right)$, for every $x \in \mathbf{R}^{3}$. This implies in particular, because of the arc-connectedness of attainable sets, that there exists $y \in \mathcal{A}(w, \mathscr{F} \lambda, w)$ such that $\|y-w\|=1$. Let $\rho<r$ and fix $z \in B(w, \rho)$. Since $\mathcal{A}\left(z,-\mathscr{F}_{\lambda, w}\right)$ is unbounded, then, again by arc-connectedness, there exists $z^{\prime} \in \mathcal{A}\left(z,-\mathscr{F}_{\lambda, w}\right)$ such that $\left\|z^{\prime}-w\right\|=r$. Since from every point of the sphere of center $w$ and radius 1 we can reach any point of the sphere of center $w$ and radius $r$, the point $z^{\prime}$ is reachable from $y$ by the family $\mathscr{F}_{\lambda, w}$. Finally, every point $z \in B(w, \rho)$ belongs to $\mathcal{A}\left(w, \mathscr{F}_{\lambda, w}\right)$ (see Fig. 1 ). Since $\mathscr{F}_{\lambda, w}$ is the transformation of $\mathscr{F}$ by a diffeomorphism preserving $w$, we deduce that $\mathcal{A}(w, \mathscr{F})$ contains a neighborhood of $w$. Therefore, every attainable set $\mathcal{A}(x, \mathscr{F})$ is open.

Since the control system defined by $-\mathscr{F}$ has the same form as that defined by $\mathscr{F}$ and verifies the hypotheses of both Lemmas 5.10 and 5.11 , the reasoning above proves also that every attainable set $\mathcal{A}(x,-\mathscr{F})$ is open. Take now $y$ in the closure of $\mathcal{A}(x, \mathscr{F})$. The set $\mathcal{A}(y,-\mathscr{F})$ being open, there exists $z \in \mathcal{A}(y,-\mathscr{F}) \cap \mathcal{A}(x, \mathscr{F})$ which proves that $y$ is reachable from $x$ by the family $\mathscr{F}$. Every attainable set $\mathcal{A}(x, \mathscr{F})$ is therefore both open and closed; thus it is equal to $\mathbf{R}^{3}$.

\subsection{Generic controllability: physical interpretation}

The scope of this section is to provide a better physical insight of Theorem 5.1. More precisely, we want to check that for $m \geq 3$ there exists an open and dense set in the (suitably defined) space of microscopic organisms modeled here such that the corresponding control system (3.1)-(3.3) is controllable.

The space of organisms, denoted by $\Sigma_{m}$, will be identified with the set of pairs $(\Omega, \Psi)$, where $\Omega \subset \mathbf{R}^{3}$ is open, nonempty, connected, bounded, and of class $\mathcal{C}^{2}$, while $\Psi$ is the $m$-uple of functions in $\mathcal{C}^{2}\left(\partial \Omega, \mathbf{R}^{3}\right)$ determining the action of the controls (see Sect. 3). For simplicity we assume that the density $\delta$ of the organism is constant, so that the inertia matrix $J$ is determined (up to the positive multiplicative constant $\delta$ ) by the shape $\Omega$. (For the general case see Rem. 5.14.) The topology on $\Sigma_{m}$ can be defined assigning the basis of open subsets defined by

$$
\begin{gathered}
\mathscr{N}_{m, \varepsilon}(\Omega, \Psi)=\left\{((\operatorname{Id}+v)(\Omega), \Phi) \mid v \in W^{3, \infty}\left(\Omega, \mathbf{R}^{3}\right), \Phi \in\left(\mathcal{C}^{2}\left(\partial \Omega, \mathbf{R}^{3}\right)\right)^{m}\right. \\
\left.\|v\|_{3, \infty}<\varepsilon,\|\Phi \circ(\operatorname{Id}+v)-\Psi\|_{\mathcal{C}^{2}(\Omega)}<\varepsilon\right\}
\end{gathered}
$$

for all $\varepsilon \in(0,1 / 2)$ and all $(\Omega, \Psi) \in \Sigma_{m}$ (see [20] or, for a different approach, [8]). By extension $\Sigma_{0}$ denotes 
the set of $\mathcal{C}^{2}$, open, nonempty, connected, bounded subsets of $\mathbf{R}^{3}$ endowed with the topology whose basis is given by all

$\varepsilon \in(0,1 / 2), \Omega \in \Sigma_{0}$.

$$
\mathscr{N}_{0, \varepsilon}(\Omega)=\left\{(\operatorname{Id}+v)(\Omega) \mid v \in W^{3, \infty}\left(\Omega, \mathbf{R}^{3}\right),\|v\|_{3, \infty}<\varepsilon\right\}
$$

Theorem 5.12. Assume that $m \geq 3$. There exists an open and dense set $\mathcal{O}$ in $\Sigma_{m}$ such that (3.1)-(3.3) is controllable if $(\Omega, \Psi)$ belongs to $\mathcal{O}$. Moreover, there exists an open and dense set $\mathcal{P}$ in $\Sigma_{0}$ such that $\mathcal{O} \cap(\{\Omega\} \times$ $\left.\left(\mathcal{C}^{2}\left(\partial \Omega, \mathbf{R}^{3}\right)\right)^{m}\right)$ is dense in $\{\Omega\} \times\left(\mathcal{C}^{2}\left(\partial \Omega, \mathbf{R}^{3}\right)\right)^{m}$ for every $\Omega$ in $\mathcal{P}$.

Proof. Denote by $F(\Omega, \Psi)$ the triple $(A, B, J)$ associated to an element $(\Omega, \Psi)$ of $\Sigma_{0}$ through (3.5), (3.7) and (3.9). We want to show that each condition appearing in the statement of Propositions 5.2 and 5.3 is satisfied by the elements of the image through $F$ of an open and dense subset of $\Sigma_{0}$.

Notice that the map $\Psi \mapsto B$ is onto when considered from $\left(\mathcal{C}^{2}\left(\partial \Omega, \mathbf{R}^{3}\right)\right)^{m}$ to $\mathscr{M}_{6 \times m}(\Omega$ fixed). Indeed, according to [19], Lemma $4.1, g^{(1)}, g^{(2)}, g^{(3)}, G^{(1)}, G^{(2)}, G^{(3)}$ are linearly independent in $L^{2}\left(\partial \Omega, \mathbf{R}^{3}\right)$. Therefore, since the orthogonal to a smooth function in $\mathcal{C}^{2}\left(\partial \Omega, \mathbf{R}^{3}\right)$ with respect to the $L^{2}$-product has infinite codimension, the relation (3.9) defines a surjective map. In particular, the pre-image of an open and dense set in $\mathscr{M}_{6 \times m}$ is open and dense in $\left(\mathcal{C}^{2}\left(\partial \Omega, \mathbf{R}^{3}\right)\right)^{m}$.

Let us now take into account the dependence on $\Omega$. We claim that the map from $\Sigma_{0}$ to $\mathbf{R}^{3}$ that associates to a domain $\Omega$ the spectrum of the corresponding inertia matrix - with eigenvalues repeated according to their multiplicity and with $\delta$ normalized to one - is locally open at every point. In order to check it, fix $\Omega \in \Sigma_{0}$ and a system of coordinates such that $0_{3}$ is the center of mass of $\Omega$ and $e_{1}, e_{2}, e_{3}$ its principal axes of inertia. Then the elements of the normalized inertia matrix $J^{0}=J / \delta$ of $\Omega$ are

$$
J_{i j}^{0}= \begin{cases} \pm \int_{\Omega} x_{i} x_{j} \mathrm{~d} x=0 & \text { if } i \neq j, \\ \int_{\Omega}\left(\sum_{k \neq i} x_{k}^{2}\right) \mathrm{d} x & \text { if } i=j .\end{cases}
$$

Apply the transformation $\operatorname{Id}+v$ to $\Omega$, where $v$ is the diagonal matrix $\operatorname{diag}\left(v_{1}, v_{2}, v_{3}\right)$, and denote by $J^{0}(v)$ the normalized inertia matrix of $(\operatorname{Id}+v)(\Omega)$. Then

$$
J_{i j}^{0}(v)= \begin{cases} \pm\left(\Pi_{k=1}^{3}\left(1+v_{k}\right)\right)\left(1+v_{i}\right)\left(1+v_{j}\right) \int_{\Omega} x_{i} x_{j} \mathrm{~d} x=0 & \text { if } i \neq j \\ \pm\left(\Pi_{k=1}^{3}\left(1+v_{k}\right)\right) \int_{\Omega}\left(\sum_{k \neq i}\left(1+v_{k}\right)^{2} x_{k}^{2}\right) \mathrm{d} x & \text { if } i=j\end{cases}
$$

The spectrum of $J^{0}(v)$ is therefore given by the components of the vector $\sigma^{0}(v)=\left( \pm\left(\Pi_{k=1}^{3}\left(1+v_{k}\right)\right) \int_{\Omega}\left(\sum_{k \neq i}(1+\right.\right.$ $\left.\left.\left.v_{k}\right)^{2} x_{k}^{2}\right) \mathrm{d} x\right)_{i=1}^{3}$. A straightforward computation shows that the determinant of the Jacobian matrix of $\sigma^{0}$ with respect to $\left(v_{1}, v_{2}, v_{3}\right)$, computed at $v_{1}=v_{2}=v_{3}=0$, is different form zero. The map $v \mapsto \sigma^{0}(v)$ is therefore a submersion at $v=0$.

In particular, the eigenvalues of $J$ are simple for $\Omega$ in an open and dense subset of $\Sigma_{0}$, independently of $\Psi$. If $b_{2}$ does not belong to any of the three planes generated by pairs of eigenvectors of $J$, then (5.2) is automatically verified.

Similarly, one notices that all the assumptions appearing in the statements of Propositions 5.2 and 5.3 can be represented in the form $G(\Omega, \Psi) \in O$ where $O$ is an open and dense subset of a finite-dimensional vector space $V$, the $\operatorname{map} G: \Sigma_{m} \rightarrow V$ is continuous and for $\Omega$ in an open and dense subset of $\Sigma_{0}$ we have that $G(\Omega, \Psi)$ belongs to $\mathcal{O}$ if $B$ belongs to an open and dense subset of $\mathscr{M}_{6 \times m}$ (possibly depending on $\Omega$ ). Therefore, $G^{-1}(\mathcal{O})$ is open and dense in $\Sigma_{m}$.

Remark 5.13. As recalled above it follows from [19] that, once $\Omega$ is fixed, the linear map associating to the $m$-uple $\Psi$ the matrix $B$ through (3.9) is onto as a map from $\left(L^{2}\left(\partial \Omega, \mathbf{R}^{3}\right)\right)^{m}$ to $\mathscr{M}_{6 \times m}$. Thanks to this result, and to the remark that (3.1)-(3.3) is controllable if $m=6$ and $B$ is invertible, the authors prove that for $m \geq 6$, for an open and dense subset of $m$-uples in $\mathcal{C}^{2}\left(\partial \Omega, \mathbf{R}^{3}\right)$ ( $\Omega$ fixed) the corresponding system is controllable (see [19], Thm. 1.1). In the language adopted here the result of [19] says that, for every fixed $\Omega$ and for $m \geq 6$, controllability is a generic property with respect to $\Psi$. 
Remark 5.14. In order to introduce a reasonable notion of genericity in the case where the density $\delta$ of the organism is not constant, it is necessary to include $\delta$ in the definition of $\Sigma_{m}$. Let $\hat{\Sigma}_{m}$ be the set of triples $(\Omega, \Psi, \delta)$ where $(\Omega, \Psi) \in \Sigma_{m}$ and $\delta \in L^{\infty}(\Omega,(0,+\infty))$ and endow $\hat{\Sigma}_{m}$ with the topology whose basis is given by all

$$
\begin{gathered}
\hat{\mathscr{N}}_{m, \varepsilon}(\Omega, \Psi, \delta)=\left\{((\operatorname{Id}+v)(\Omega), \Phi, \gamma) \mid v \in W^{3, \infty}\left(\Omega, \mathbf{R}^{3}\right), \Phi \in\left(\mathcal{C}^{2}\left(\partial \Omega, \mathbf{R}^{3}\right)\right)^{m}, \gamma \in L^{\infty}(\Omega,(0,+\infty)),\right. \\
\left.\|v\|_{3, \infty}<\varepsilon,\|\Phi \circ(\operatorname{Id}+v)-\Psi\|_{\mathcal{C}^{2}(\Omega)}<\varepsilon,\|\gamma \circ(\operatorname{Id}+v)-\delta\|_{L^{\infty}(\Omega)}<\varepsilon\right\},
\end{gathered}
$$

$\varepsilon \in(0,1 / 2),(\Omega, \Psi, \delta) \in \hat{\Sigma}_{m}$. Following the same arguments of proof as above, one can prove that if $m \geq 3$ there exists an open and dense set $\mathcal{O}$ in $\hat{\Sigma}_{m}$ such that (3.1)-(3.3) is controllable if $(\Omega, \Psi, \delta)$ belongs to $\mathcal{O}$. The only difference consists in verifying that the map associating to $(\Omega, \delta)$ the spectrum of $J$ is locally open, and this can be done by taking the same perturbation $\left(\operatorname{Id}+\operatorname{diag}\left(v_{1}, v_{2}, v_{3}\right)\right)(\Omega)$ as above for $\Omega$ and the perturbation $\delta \circ\left(\operatorname{Id}+\operatorname{diag}\left(v_{1}, v_{2}, v_{3}\right)\right)$ for $\delta$.

\section{SWIMMING SPHERICAL MICROSCOPIC ORGANISMS}

When the microscopic organism happens to be spherical, the equations presented in the previous sections have a very special form. As described in [19], general results in hydrodynamics (see, e.g., [11]) show that the matrix $A$ corresponding to a spherical organism is diagonal and, more precisely, of the form

$$
A=\left(\begin{array}{cc}
-\rho_{1} \mathrm{Id} & 0 \\
0 & -\rho_{2} \mathrm{Id}
\end{array}\right)
$$

with

$$
\rho_{2}>\rho_{1}>0 .
$$

Moreover, since the inertia matrix $J$ is proportional to the identity (assuming that the mass distribution is homogeneous inside $\Omega$ ), the nonlinear term $E$ appearing in (3.1) is given by

$$
E(z)=\left(\begin{array}{c}
\omega \times \xi \\
0_{3}
\end{array}\right)
$$

Notice that in the spherical case $\omega$ satisfies a well-defined control subsystem, i.e.,

$$
\dot{\omega}=-\rho_{2} \omega+B_{2} u .
$$

We are going to prove the following result.

Theorem 6.1. Let $\Omega$ be a ball and assume that the mass distribution of $\Omega$ is homogeneous. Then the control system (3.1)-(3.3) is controllable if and only if the rank of $B_{2}$ is equal to three and $B_{1}$ is different from $0_{3 \times m}$.

The easier part of the proof is to show that the controllability of (3.1)-(3.3) implies that

$$
\operatorname{rank} B_{2}=3, \quad B_{1} \neq 0_{3 \times m} .
$$

Indeed, the linear control system (6.2) is, as it is well known, controllable if and only if $\operatorname{rank} B_{2}=3$. Moreover, if $B_{1}=0_{3 \times m}$, then the space $\left\{0_{3}\right\} \times \mathbf{R}^{3}$ is invariant for the dynamics of the control system (3.1), which is therefore non-controllable.

The converse implication will be proven in several steps. 


\subsection{The case where $B_{1}$ and $B_{2}$ are linearly independent}

In this section we study the case where the organism is spherical and

$$
\operatorname{rank} B_{2}=3, \quad B_{1} \notin \operatorname{span}\left(B_{2}\right) .
$$

Let us prove the following technical result.

Lemma 6.2. If assumption (6.4) holds true, then there exist $c_{1}, c_{2}, c_{3} \in \mathbf{R}^{3}$, an orthonormal basis $\left\{d_{1}, d_{2}, d_{3}\right\}$ of $\mathbf{R}^{3}$, and a $m \times 3$ matrix $\Gamma$ such that

$$
B_{1} \Gamma v=\sum_{i=1}^{3} v_{i} c_{i}, \quad B_{2} \Gamma v=\sum_{i=1}^{3} v_{i} d_{i}, \quad c_{1} \times d_{1} \neq 0_{3}
$$

Proof. Firstly, let us consider the case $m=3$. Then $B_{2}$ is invertible and for every orthonormal basis $\left\{d_{1}, d_{2}, d_{3}\right\}$ of $\mathbf{R}^{3}$, we have

$$
B_{2} \Gamma v=\sum_{i=1}^{3} v_{i} d_{i}
$$

for $\Gamma=B_{2}^{-1}\left(d_{1}\left|d_{2}\right| d_{3}\right)$, where $\left(d_{1}\left|d_{2}\right| d_{3}\right)$ denotes the matrix whose columns are $d_{1}, d_{2}$ and $d_{3}$. Since $B_{1} B_{2}^{-1}$ is not proportional to Id, then $d_{1}$ can be chosen such that $d_{1} \times\left(B_{1} B_{2}^{-1} d_{1}\right) \neq 0_{3}$. It suffices to complete $d_{1}$ to any orthonormal basis $\left\{d_{1}, d_{2}, d_{3}\right\}$, since $B_{1} \Gamma v=\sum_{i=1}^{3} v_{i} c_{i}$ with $c_{i}=B_{1} B_{2}^{-1} d_{i}$.

Let now $m>3$. Denote by $B_{i}^{(j)}$ the $j$ th column of $B_{i}$.

The second case we consider is when there exist $1 \leq j_{1}, j_{2}, j_{3} \leq m$ such that $B_{2}^{\left(j_{1}\right)}, B_{2}^{\left(j_{2}\right)}, B_{2}^{\left(j_{3}\right)}$ are linearly independent and $\left(B_{1}^{\left(j_{1}\right)}\left|B_{1}^{\left(j_{2}\right)}\right| B_{1}^{\left(j_{3}\right)}\right) \notin \operatorname{span}\left(B_{2}^{\left(j_{1}\right)}\left|B_{2}^{\left(j_{2}\right)}\right| B_{2}^{\left(j_{3}\right)}\right)$. Consider the $m \times 3$ matrix $\Gamma^{\prime}$ whose all entries are zero excepted the entries $\left(j_{1}, 1\right),\left(j_{2}, 2\right)$ and $\left(j_{3}, 3\right)$ that we take equal to 1 . The columns of the $3 \times 3$ matrices $B_{i}^{\prime}=B_{i} \Gamma^{\prime}$ are $B_{i}^{\left(j_{1}\right)}, B_{i}^{\left(j_{2}\right)}$ and $B_{i}^{\left(j_{3}\right)}(i=1,2)$. The matrices $B_{1}^{\prime}$ and $B_{2}^{\prime}$ fulfill the hypotheses of the case $m=3$ and we are done.

Finally, consider the case in which for every triple $1 \leq j_{1}, j_{2}, j_{3} \leq m$ such that $B_{2}^{\left(j_{1}\right)}, B_{2}^{\left(j_{2}\right)}, B_{2}^{\left(j_{3}\right)}$ are linearly independent, we have that $\left(B_{1}^{\left(j_{1}\right)}\left|B_{1}^{\left(j_{2}\right)}\right| B_{1}^{\left(j_{3}\right)}\right)$ is proportional to $\left(B_{2}^{\left(j_{1}\right)}\left|B_{2}^{\left(j_{2}\right)}\right| B_{2}^{\left(j_{3}\right)}\right)$. Let

$$
\left\{j_{1}, \ldots, j_{k}\right\}=\left\{j \mid 1 \leq j \leq m, B_{2}^{(j)} \neq 0_{3}\right\}
$$

Then $\left(B_{1}^{\left(j_{1}\right)}|\ldots| B_{1}^{\left(j_{k}\right)}\right)$ is proportional to $\left(B_{2}^{\left(j_{1}\right)}|\ldots| B_{2}^{\left(j_{k}\right)}\right)$. Assumption (6.4) implies that there exists $1 \leq j \leq m$ such that $B_{1}^{(j)} \neq 0_{3}$ and $B_{2}^{(j)}=0_{3}$. The column $B_{1}^{(j)}$ cannot be proportional to both columns $B_{2}^{\left(j_{1}\right)}$ and $B_{2}^{\left(j_{2}\right)}$ so there exists an index, say $j_{1}$, such that $B_{1}^{(j)}$ is not proportional to $B_{2}^{\left(j_{1}\right)}$, this implies that $B_{1}^{\left(j_{1}\right)}+B_{1}^{(j)}$ is not proportional to $B_{2}^{\left(j_{1}\right)}$ (since $B_{1}^{\left(j_{1}\right)}$ and $B_{2}^{\left(j_{1}\right)}$ are proportional). Consider the $m \times 3$ matrix $\Gamma^{\prime}$ whose all entries are 0 excepted the entries $(j, 1),\left(j_{1}, 1\right),\left(j_{2}, 2\right)$ and $\left(j_{3}, 3\right)$ that we take equal to 1 . The columns of the $3 \times 3$ matrix $B_{1}^{\prime}=B_{1} \Gamma^{\prime}$ are $B_{1}^{(j)}+B_{1}^{\left(j_{1}\right)}, B_{1}^{\left(j_{2}\right)}$ and $B_{1}^{\left(j_{3}\right)}$ while the columns of $B_{2}^{\prime}=B_{2} \Gamma^{\prime}$ are $B_{2}^{\left(j_{1}\right)}, B_{2}^{\left(j_{2}\right)}$ and $B_{2}^{\left(j_{3}\right)}$. The matrices $B_{1}^{\prime}$ and $B_{2}^{\prime}$ fulfill the hypotheses of the case $m=3$ and we are done.

Notice now that for every $Q \in \mathrm{SO}(3)$, the change of coordinates $\xi^{\prime}=Q \xi, \omega^{\prime}=Q \omega, \zeta^{\prime}=\zeta, R^{\prime}=R Q^{-1}$ preserves the dynamics of (3.1)-(3.3). This is equivalent to say that, up to a change of coordinates, we can replace $\left(B_{1}, B_{2}\right)$ by $\left(Q B_{1}, Q B_{2}\right)$, for every choice of $Q \in \mathrm{SO}(3)$. Let $d_{1}, d_{2}, d_{3}$ be as in (6.5) and choose $Q$ such that $Q d_{i}=e_{i}$ for $i=1,2,3$ (where we recall that $\left(e_{i}\right)_{i=1,2,3}$ denotes the canonical basis in $\mathbf{R}^{3}$ ). The above transformations show that proving the controllability of the control system (3.1)-(3.3) under assumption (6.4) is equivalent to proving it under the hypotheses that $m=3, B_{2}=\mathrm{Id}$, and that the first column of $B_{1}$ is not proportional to $e_{1}$.

Denote by $b_{1}, b_{2}, b_{3}$ the columns of $B_{1}$. Accordingly, the controlled vector fields are the three constant vector fields $X_{i}=\left(b_{i}, e_{i}, 0_{6}\right), i=1,2,3$. 
Lemma 6.3. If (6.4) holds then system (3.1)-(3.3) is Lie-bracket generating.

Proof. Applying (3.4) in the case $J=\mathrm{Id}$ we obtain that for every $v_{1}, v_{2} \in \mathbf{R}^{3}$ the constant vector field $V=\left(v_{1}, v_{2}, 0_{6}\right)$ satisfies

$$
\left[\left[X_{0}, X_{i}\right], V\right]=\left(e_{i} \times v_{1}-b_{i} \times v_{2}, 0_{9}\right) .
$$

In particular, taking $i=1$ and $V=X_{1}$ in (6.6), we deduce that $Z=\left(e_{1} \times b_{1}, 0_{9}\right)$ belongs to $\mathscr{L}$. Applying again (6.6) to $V=Z$ we get that, for $i=1,2,3, Z_{i}=\left(e_{i} \times\left(e_{1} \times b_{1}\right), 0_{9}\right)$ belongs to $\mathscr{L}$. Since $Z, Z_{1}, Z_{2}$, and $Z_{3}$ span $\mathbf{R}^{3} \times\left\{0_{9}\right\}$, it follows that $\mathscr{L}$ contains

$$
\operatorname{span}\left(X_{1}, X_{2}, X_{3}\right)+\mathbf{R}^{3} \times\left\{0_{9}\right\}=\mathbf{R}^{6} \times\left\{0_{6}\right\}
$$

As noticed in Remark 3.1 this proves the lemma.

The controllability of system (3.1) follows from Proposition 5.3: indeed, $\tilde{A}_{11}=\rho_{1} \operatorname{Id}$ is symmetric and invertible and hypothesis (6.4) implies that $B_{1}=B_{1} B_{2}^{-1}$ has a smaller set of eigenvectors than $J=$ Id. Theorem 6.1 is therefore proven, because of Proposition 4.1, under the stronger assumption that (6.4) holds true.

\subsection{The proportional case}

In order to complete the proof of Theorem 6.1, we have to prove that system (3.1)-(3.3) is controllable when

$$
\operatorname{rank} B_{2}=3, \quad B_{1}=\lambda B_{2}, \quad \lambda \neq 0
$$

Using the same normalization argument as in the previous section, we can assume that $m=3$ and $B_{2}=\mathrm{Id}$. Moreover, we can take $\lambda=1$ by performing the change of coordinates $\xi^{\prime}=\xi / \lambda, \omega^{\prime}=\omega, \zeta^{\prime}=\zeta / \lambda, R^{\prime}=R$. Therefore, without loss of generality, $X_{i}=\left(e_{i}, e_{i}, 0_{6}\right)$ for $i=1,2,3$.

Let us prove the Lie-bracket generating condition. We have

$$
\left[X_{0}, X_{i}\right]=\left(-\rho_{1} e_{i},-\rho_{2} e_{i}, R e_{i}, R S\left(e_{i}\right)\right)
$$

and

A further computation yields

$$
\left[\left[X_{0}, X_{i}\right],\left[X_{0}, X_{j}\right]\right]=\left(0_{6}, 2 R\left(e_{j} \times e_{i}\right), R S\left(e_{j} \times e_{i}\right)\right) .
$$

$$
\left[\left[\left[X_{0}, X_{i}\right],\left[X_{0}, X_{j}\right]\right],\left[X_{0}, X_{k}\right]\right]=\left(0_{6}, 3 R\left(e_{k} \times\left(e_{j} \times e_{i}\right)\right), R S\left(e_{k} \times\left(e_{j} \times e_{i}\right)\right)\right) .
$$

Therefore, the Lie algebra $\mathscr{L}$ contains the following vector fields

$$
\left(\begin{array}{c}
e_{i} \\
e_{i} \\
0_{3} \\
0_{3}
\end{array}\right), \quad\left(\begin{array}{c}
-\rho_{1} e_{j} \\
-\rho_{2} e_{j} \\
R e_{j} \\
R S\left(e_{j}\right)
\end{array}\right), \quad\left(\begin{array}{c}
0_{3} \\
0_{3} \\
2 R e_{k} \\
R S\left(e_{k}\right)
\end{array}\right), \quad\left(\begin{array}{c}
0_{3} \\
0_{3} \\
3 R e_{l} \\
R S\left(e_{l}\right)
\end{array}\right),
$$

for $i, j, k, l=1,2,3$. As a consequence, $\mathscr{L}$ contains also

$$
\left(\begin{array}{c}
\left(\rho_{2}-\rho_{1}\right) e_{i} \\
0_{3} \\
R e_{i} \\
R S\left(e_{i}\right)
\end{array}\right), \quad\left(\begin{array}{c}
0_{3} \\
\left(\rho_{1}-\rho_{2}\right) e_{j} \\
R e_{j} \\
R S\left(e_{j}\right)
\end{array}\right), \quad\left(\begin{array}{c}
0_{3} \\
0_{3} \\
R e_{k} \\
0_{3}
\end{array}\right), \quad\left(\begin{array}{c}
0_{3} \\
0_{3} \\
0_{3} \\
R S\left(e_{l}\right)
\end{array}\right)
$$

for $i, j, k, l=1,2,3$. These vector fields form a moving frame on the manifold $\mathbf{R}^{9} \times \operatorname{SO}(3)\left(\right.$ since $\left.\rho_{1} \neq \rho_{2}\right)$. 
In order to conclude the proof of Theorem 6.1 we need to show that (3.1) is controllable. Applying Lemma 5.5 this turns out to be equivalent to the controllability of the following system,

$$
\dot{x}=-\rho_{1} x-v \times x+\left(\rho_{2}-\rho_{1}\right) v, \quad x \in \mathbf{R}^{3},
$$

with the control $v$ taking values in $\mathbf{R}^{3}$. Decomposing the control as $v=w x+W$ with $w \in \mathbf{R}$ and $W$ orthogonal to $x$, equation (6.8) rewrites as

$$
\dot{x}=\left(-\rho_{1}+\left(\rho_{2}-\rho_{1}\right) w\right) x-W \times x .
$$

Clearly, choosing $W=0_{3}$ allows to move as desired along $\operatorname{span}(x)$, while the choice $w=\rho_{1} /\left(\rho_{2}-\rho_{1}\right)$ permits to attain the sphere of radius $\|x\|$ centered at the origin. The controllability of (3.1) is thus proven and the proof of Theorem 6.1 completed.

\section{The CASE Where the Densities of THE MiCROSCOPIC ORGANisM AND OF THE FLUid HAVE THE SAME ORDER OF MAGNITUDE}

As pointed out in [19], Section 2, the assumption that the densities of the microscopic organism and of the fluid have the same order of magnitude leads to stronger simplifications than in the general case studied in the previous sections. In particular, according to the remarks of page 6 the control system (2.18)-(2.27) presented in [19] reduces in this case to

$$
\begin{aligned}
-\Delta v+\nabla p=0 & \text { on } F \times(0, T), \\
\operatorname{div} v=0 & \text { on } F \times(0, T), \\
v(y, t)=\xi(t)+\omega(t) \times y+\sum_{i=1}^{m} u_{i}(t) \psi_{i}(y) & \text { for }(y, t) \in \partial \Omega \times(0, T), \\
\lim _{|y| \rightarrow \infty} v(y, t)=0 & \text { for } t \in(0, T), \\
\int_{\partial \Omega} \sigma(v, p) n \mathrm{~d} s=0 & \text { on }(0, T), \\
\int_{\partial \Omega} y \times \sigma(v, p) n \mathrm{~d} s=0 & \text { on }(0, T), \\
\dot{\zeta}=R \xi & \text { on }(0, T), \\
\dot{R}=R S(\omega) & \text { on }(0, T) .
\end{aligned}
$$

Physically, $v$ represents the field of velocities of the fluid in $F$ (the coordinates are attached to the body), $p$ is the pressure, $\xi$ and $\omega$ are the linear and angular velocities of the organism and finally $\zeta$ and $R$ give its position and orientation with respect to a fixed frame. Recall that $\psi_{1}, \ldots, \psi_{m}$ are the functions characterizing the control actions and that $\sigma$ is the Cauchy stress defined in (3.6).

Given a time-interval $[0, T]$ and a control $u \in L^{\infty}\left([0, T], \mathbf{R}^{m}\right)$ there exists a unique solution $(v, p, \xi, \omega, \zeta, R)$ of (7.1)-(7.8) satisfying

$$
\begin{gathered}
v \in H^{1}\left([0, T], L^{s}(F) \cap D^{1, r}(F) \cap D^{2, \theta}(F) \cap \mathcal{C}^{\infty}(F)\right) \\
\sup _{y \in F}(1+\|y\|)\|v(y, t)\|<\infty \quad \text { for almost every } t \in[0, T] \\
p \in H^{1}\left([0, T], L^{r}(F) \cap D^{1, \theta}(F) \cap \mathcal{C}^{\infty}(F)\right), \\
\xi \in H^{1}\left([0, T], \mathbf{R}^{3}\right), \omega \in H^{1}\left([0, T], \mathbf{R}^{3}\right), \zeta \in \mathcal{C}^{1}\left([0, T], \mathbf{R}^{3}\right), \quad R \in \mathcal{C}^{1}([0, T], \operatorname{SO}(3)),
\end{gathered}
$$

for $s \in(3, \infty], r \in(3 / 2, \infty]$ and $\theta \in(1, \infty)$ (see $[9,19])$. 
The finite-dimensional reduction of system (7.1)-(7.8) can be obtained following the procedure proposed in [19]. To this extent, as in Section 3, define the fundamental solutions $\left(h^{(i)}, p^{(i)}\right)$ and $\left(H^{(i)}, P^{(i)}\right)$ of the Stokes system and associate to them the matrices $\Theta^{k}, \Upsilon^{k}, \kappa, \mathcal{K}$. Let $L$ be the $n \times m$ matrix

$$
L=-\left(\begin{array}{cc}
\Theta^{1} & \Theta^{2} \\
\Upsilon^{1} & \Upsilon^{2}
\end{array}\right)^{-1}\left(\begin{array}{c}
\kappa \\
\mathcal{K}
\end{array}\right)
$$

Lemma 7.1. For almost every $t \in(0, T)$ we have

$$
\left(\begin{array}{c}
\xi(t) \\
\omega(t)
\end{array}\right)=L u(t)
$$

Proof. According to [19], Lemma 3.4, we have

$$
\begin{gathered}
\int_{\partial \Omega} g^{(i)} \cdot v \mathrm{~d} s=\left[\int_{\partial \Omega} \sigma(v, p) n \mathrm{~d} s\right]_{i}=0 \\
\int_{\partial \Omega} G^{(i)} \cdot v \mathrm{~d} s=\left[\int_{\partial \Omega} y \times \sigma(v, p) n \mathrm{~d} s\right]_{i}=0
\end{gathered}
$$

where the equalities in the right-hand sides follow from (7.5) and (7.6).

On the other hand, for almost every $t \in(0, T)$,

$$
\int_{\partial \Omega} g^{(i)} \cdot v \mathrm{~d} s=\int_{\partial \Omega} g^{(i)} \cdot\left(\xi+\omega \times y+\sum_{j=1}^{m} u_{j} \psi_{j}\right) \mathrm{d} s=-\left(\Theta^{1} \xi+\Theta^{2} \omega+\kappa u\right)_{i},
$$

and, similarly

Therefore,

$$
\int_{\partial \Omega} G^{(i)} \cdot v \mathrm{~d} s=-\left(\Upsilon^{1} \xi+\Upsilon^{2} \omega+\mathcal{K} u\right)_{i}
$$

$$
\left(\begin{array}{c}
\kappa \\
\mathcal{K}
\end{array}\right) u=-\left(\begin{array}{cc}
\Theta^{1} & \Theta^{2} \\
\Upsilon^{1} & \Upsilon^{2}
\end{array}\right)\left(\begin{array}{c}
\xi \\
\omega
\end{array}\right)
$$

which proves the lemma.

System (7.1)-(7.8) therefore reduces to the control system

$$
\left\{\begin{aligned}
\dot{\zeta} & =R L^{1} u, \\
\dot{R} & =R S\left(L^{2} u\right), \quad u \in \mathbf{R}^{m},
\end{aligned}\right.
$$

where $L^{1}, L^{2} \in \mathscr{M}_{3 \times m}$ are such that

$$
L=\left(\begin{array}{c}
L^{1} \\
L^{2}
\end{array}\right)
$$

By homogeneity, since the set of admissible controls is the whole $\mathbf{R}^{m}$, the controllability in arbitrary small time of system (7.9) is equivalent to the controllability of the control system whose admissible velocities are given by the family of vector fields $\mathcal{F}=\left\{ \pm X_{i} \mid 1 \leq i \leq m\right\}$ defined by

$$
X_{i}(\zeta, R)=\left(R b_{i}, R S\left(c_{i}\right)\right)
$$

where the $b_{i}$ 's denote the columns of $L^{1}$ and the $c_{i}$ 's those of $L^{2}$. Moreover, since $\mathcal{F}$ is symmetrical, the controllability of this family is equivalent to the fact that the Lie algebra generated by $\mathcal{F}$, denoted by $\mathscr{L}$, is of rank 6 at every point of $\mathbf{R}^{3} \times \mathrm{SO}(3)$ (see, e.g., [1], Cor. 5.11). 
Theorem 7.2. The control system (7.9) is controllable in arbitrary small time if and only if one of the following conditions is satisfied:

(1) $\operatorname{rank}\left(L^{2}\right) \geq 2$ and $\left(L^{1}\right)^{\mathrm{T}} L^{2}+\left(L^{2}\right)^{\mathrm{T}} L^{1} \neq 0_{m \times m}$;

(2) $\operatorname{rank}\left(L^{2}\right)=2$ and $\operatorname{rank}(L) \geq 3$.

Proof. First of all notice that the Lie bracket between two vector fields of the form $Z_{i}(\zeta, R)=\left(R v_{i}, R S\left(w_{i}\right)\right)$, $i=1,2$, is given by

$$
\left[Z_{1}, Z_{2}\right](\zeta, R)=\left(R\left(w_{1} \times v_{2}-w_{2} \times v_{1}\right), R S\left(w_{1} \times w_{2}\right)\right) .
$$

Let us prove the "if" part of the theorem. Suppose that we can find in $\mathscr{L}$ two vector fields $Y_{1}, Y_{2}$ of the form

$$
Y_{i}(\zeta, R)=\left(R \beta_{i}, R S\left(\gamma_{i}\right)\right), \quad i=1,2,
$$

such that the vectors $\gamma_{1}$ and $\gamma_{2}$ are linearly independent and that the inner product $\left\langle\beta_{1}, \gamma_{1}\right\rangle$ is nonzero. We prove here below that, in this case, the Lie algebra $\mathscr{L}$ is of full rank at every point of $\mathbf{R}^{3} \times \mathrm{SO}(3)$. Then we show that both (1) and (2). guarantee that, without loss of generality, such $Y_{1}$ and $Y_{2}$ can be found.

Let $Y_{3}=\left[Y_{1}, Y_{2}\right]$ whose expression can be obtained using (7.11). Define the vector field $W_{1}$ as the bracket between $Y_{3}$ and $Y_{1}$. Applying again (7.11) we obtain that

$$
W_{1}(\zeta, R)=\left[Y_{3}, Y_{1}\right](\zeta, R)=\left(\begin{array}{c}
R\left(2\left\langle\beta_{1}, \gamma_{1}\right\rangle \gamma_{2}-\left(\left\langle\beta_{1}, \gamma_{2}\right\rangle+\left\langle\beta_{2}, \gamma_{1}\right\rangle\right) \gamma_{1}-\left\langle\gamma_{1}, \gamma_{2}\right\rangle \beta_{1}+\left\|\gamma_{1}\right\|^{2} \beta_{2}\right) \\
R S\left(-\left\langle\gamma_{1}, \gamma_{2}\right\rangle \gamma_{1}+\left\|\gamma_{1}\right\|^{2} \gamma_{2}\right)
\end{array}\right)
$$

Let $W_{2}$ be the vector field obtained adding $\left\langle\gamma_{1}, \gamma_{2}\right\rangle Y_{1}-\left\|\gamma_{1}\right\|^{2} Y_{2}$ to $W_{1}$, i.e.,

$$
W_{2}(\zeta, R)=\left(\begin{array}{c}
R\left(2\left\langle\beta_{1}, \gamma_{1}\right\rangle \gamma_{2}-\left(\left\langle\beta_{1}, \gamma_{2}\right\rangle+\left\langle\beta_{2}, \gamma_{1}\right\rangle\right) \gamma_{1}\right) \\
0_{3}
\end{array}\right)
$$

Since $W_{2}$ is in $\mathscr{L}$, then the vector field $Y_{4}=\left[W_{2}, Y_{1}\right]$ belongs to $\mathscr{L}$ as well and we have that $Y_{4}(\zeta, R)=$ $\left(R\left(2\left\langle\beta_{1}, \gamma_{1}\right\rangle \gamma_{1} \times \gamma_{2}\right), 0_{3}\right)$. Consider now the vector fields $Y_{5}=\left[Y_{4}, Y_{1}\right]$ and $Y_{6}=\left[Y_{4}, Y_{2}\right]$, both belonging to $\mathscr{L}$, and whose expressions are

$$
\begin{aligned}
& Y_{5}(\zeta, R)=\left(R\left(2\left\langle\beta_{1}, \gamma_{1}\right\rangle \gamma_{1} \times\left(\gamma_{1} \times \gamma_{2}\right)\right), 0_{3}\right) \\
& Y_{6}(\zeta, R)=\left(R\left(2\left\langle\beta_{1}, \gamma_{1}\right\rangle \gamma_{2} \times\left(\gamma_{1} \times \gamma_{2}\right)\right), 0_{3}\right)
\end{aligned}
$$

Since each matrix $R$ in $\mathrm{SO}(3)$ is invertible, and because $\left\langle\beta_{1}, \gamma_{1}\right\rangle \neq 0$, the vector fields $Y_{1}, \ldots, Y_{6} \operatorname{span} T_{(\zeta, R)} \mathbf{R}^{3} \times$ $\mathrm{SO}(3)$ at every $(\zeta, R) \in \mathbf{R}^{3} \times \mathrm{SO}(3)$ if and only if the matrix

$$
\Delta=\left(\begin{array}{cccccc}
\beta_{1} & \beta_{2} & \gamma_{2} \times \beta_{1}-\gamma_{1} \times \beta_{2} & \gamma_{1} \times \gamma_{2} & \gamma_{1} \times\left(\gamma_{1} \times \gamma_{2}\right) & \gamma_{2} \times\left(\gamma_{1} \times \gamma_{2}\right) \\
\gamma_{1} & \gamma_{2} & \gamma_{2} \times c_{1} & 0_{3} & 0_{3} & 0_{3}
\end{array}\right)
$$

is full-rank. The non-degeneracy of $\Delta$ follows easily from the assumption that the vectors $\gamma_{1}$ and $\gamma_{2}$ are linearly independent.

We shall prove now that the hypotheses (1) and (2) imply the existence of two vector fields $Y_{1}$ and $Y_{2}$ as above.

Let $r=\operatorname{rank}\left(L^{2}\right)$. Notice that for every matrix $\Gamma \in G L(m)$ the reparameterization $u \rightarrow \Gamma u$ transforms the matrix $\left(L^{1}, L^{2}\right)$ into $\left(L^{1} \Gamma, L^{2} \Gamma\right)$. The condition $\left(L^{1}\right)^{\mathrm{T}} L^{2}+\left(L^{2}\right)^{\mathrm{T}} L^{1} \neq 0_{m \times m}$ is preserved by this transformation, since, $\left(L^{1} \Gamma\right)^{\mathrm{T}} L^{2} \Gamma+\left(L^{2} \Gamma\right)^{\mathrm{T}} L^{1} \Gamma=\Gamma^{T}\left(\left(L^{1}\right)^{\mathrm{T}} L^{2}+\left(L^{2}\right)^{\mathrm{T}} L^{1}\right) \Gamma$. Thus, without loss of generality, we can assume that $L^{2}=\left(L^{2,1} \mid 0_{3 \times(m-r)}\right)$ where $L^{2,1} \in \mathscr{M}_{3 \times r}$ is of rank $r$. Let us write $L^{1}$ as $\left(L^{1,1} \mid L^{1,2}\right)$ where $L^{1,1} \in \mathscr{M}_{3 \times r}$ and $L^{1,2} \in \mathscr{M}_{3 \times(m-r)}$.

Suppose that (1) holds. If $\left(L^{1,1}\right)^{\mathrm{T}} L^{2,1}+\left(L^{2,1}\right)^{\mathrm{T}} L^{1,1} \neq 0_{r \times r}$, then we can find $1 \leq j_{1}, j_{2} \leq r$ such that $\left\langle b_{j_{1}}, c_{j_{1}}\right\rangle \neq 0$, or $\left\langle b_{j_{2}}, c_{j_{2}}\right\rangle \neq 0$, or $\left\langle b_{j_{1}}, c_{j_{2}}\right\rangle+\left\langle b_{j_{2}}, c_{j_{1}}\right\rangle \neq 0$. If $\left\langle b_{j_{1}}, c_{j_{1}}\right\rangle=\left\langle b_{j_{2}}, c_{j_{2}}\right\rangle=0$, then the inner product 
$\left\langle b_{j_{1}}+b_{j_{2}}, c_{j_{1}}+c_{j_{2}}\right\rangle=\left\langle b_{j_{1}}, c_{j_{2}}\right\rangle+\left\langle b_{j_{2}}, c_{j_{1}}\right\rangle$ is nonzero, and thus the vector fields $Y_{1}=X_{j_{1}}+X_{j_{2}}$ and $Y_{2}=X_{j_{2}}$ fulfill the required conditions. Otherwise, $\left(L^{2,1}\right)^{\mathrm{T}} L^{1,2} \neq 0_{r \times(m-r)}$ and we can fix $1 \leq j_{1}, j_{2} \leq r$ and $j_{3}>r$ such that $j_{1} \neq j_{2},\left\langle b_{j_{1}}, c_{j_{1}}\right\rangle=0$ and $\left\langle b_{j_{3}}, c_{j_{1}}\right\rangle \neq 0$. Then $Y_{1}=X_{j_{1}}+X_{j_{3}}$ and $Y_{2}=X_{j_{2}}$ fulfill the required conditions.

Assume now that (2) holds and (1) does not. Then $c_{1}$ and $c_{2}$ are linearly independent and

$$
\left\langle b_{1}, c_{1}\right\rangle=\left\langle b_{2}, c_{2}\right\rangle=\left\langle b_{1}, c_{2}\right\rangle+\left\langle b_{2}, c_{1}\right\rangle=0 .
$$

Moreover, there exists $j>r=2$ such that $b_{j} \neq 0_{3}$ and $\left\langle b_{j}, c_{1}\right\rangle=\left\langle b_{j}, c_{2}\right\rangle=0$. Therefore, there exists a real number $\alpha \neq 0$ such that $b_{3}=\alpha\left(c_{1} \times c_{2}\right)$. Then, according to (7.11),

$$
\left[X_{1}, X_{j}\right](\zeta, R)=\left(R\left(\alpha c_{1} \times\left(c_{1} \times c_{2}\right)\right), 0_{3}\right) .
$$

As $\left\langle c_{2}, c_{1} \times\left(c_{1} \times c_{2}\right)\right\rangle \neq 0$ the vector fields $Y_{1}=X_{1}$ and $Y_{2}=X_{2}+\left[X_{1}, X_{j}\right]$ fulfill the required conditions.

Let us prove now the "only if" part of the statement. First notice that if $r \leq 1$ then there exists a vector $x_{0} \in \mathbf{R}^{3} \backslash\left\{0_{3}\right\}$ such that $L^{2} u \times x_{0}=0$ for every control $u$. Multiplying equation (7.9) by $x_{0}$, we get that $\dot{R} x_{0}=0$ and so $R(t) x_{0}=R(0) x_{0}$ for every time $t$. Therefore, system (7.9) is not controllable.

Assume now that $r=\operatorname{rank}(L)=2$ and $\left(L^{1}\right)^{\mathrm{T}} L^{2}+\left(L^{2}\right)^{\mathrm{T}} L^{1}=0_{m \times m}$. Let $Y=\left[X_{1}, X_{2}\right]$, whose expression, according to (7.11), is given by

$$
Y(\zeta, R)=\left(R\left(c_{1} \times b_{2}-c_{2} \times b_{1}\right), R S\left(c_{1} \times c_{2}\right)\right) .
$$

Since $\left\langle b_{i}, c_{i}\right\rangle=0$ for $i=1,2$ and $\left\langle b_{1}, c_{2}\right\rangle+\left\langle b_{2}, c_{1}\right\rangle=0$, we obtain that

$$
\begin{aligned}
& {\left[Y, X_{1}\right]=-\left\langle c_{1}, c_{2}\right\rangle X_{1}+\left\|c_{1}\right\|^{2} X_{2}} \\
& {\left[Y, X_{2}\right]=-\left\|c_{2}\right\|^{2} X_{1}+\left\langle c_{1}, c_{2}\right\rangle X_{2} .}
\end{aligned}
$$

Therefore, the Lie algebra $\mathscr{L}$, which is generated by $X_{1}$ and $X_{2}$, is equal to the linear space of vector fields spanned by $X_{1}, X_{2}$ and $Y$ and cannot be of full rank.

Let now $r=3$ and $\left(L^{1}\right)^{\mathrm{T}} L^{2}+\left(L^{2}\right)^{\mathrm{T}} L^{1}=0_{m \times m}$. The condition $\left(L^{2,1}\right)^{\mathrm{T}} L^{1,2}=0_{3 \times(m-3)}$ implies that the columns of $L^{1,2}$ are orthogonal to all the elements of a basis of $\mathbf{R}^{3}$. Therefore, $L^{1,2}=0_{3 \times(m-3)}$. As for the columns of $L^{1,1}$, we easily obtain from $\left(L^{1,1}\right)^{\mathrm{T}} L^{2,1}+\left(L^{2,1}\right)^{\mathrm{T}} L^{1,1}=0_{3 \times 3}$ that

$$
\begin{aligned}
& b_{1}=-\alpha_{12} c_{2}-\alpha_{13} c_{3}, \\
& b_{2}=\alpha_{12} c_{1}-\alpha_{23} c_{3}, \\
& b_{3}=\alpha_{13} c_{1}+\alpha_{23} c_{2},
\end{aligned}
$$

for some $\alpha_{12}, \alpha_{13}, \alpha_{23} \in \mathbf{R}$. Without loss of generality we can assume that $\left(c_{1}, c_{2}, c_{3}\right)$ is a positively oriented orthonormal basis and an easy computation gives

$$
\begin{aligned}
& {\left[X_{1}, X_{2}\right]=X_{3},} \\
& {\left[X_{1}, X_{3}\right]=-X_{2},} \\
& {\left[X_{2}, X_{3}\right]=X_{1},}
\end{aligned}
$$

which proves that $\mathscr{L}$ is equal to the linear space of vector fields spanned by $X_{1}, X_{2}$ and $X_{3}$ and cannot be of full rank.

Acknowledgements. It is a pleasure for us to thank M. Tucsnak for fruitful discussions while preparing this manuscript. 


\section{REFERENCES}

[1] A.A. Agrachev and Y.L. Sachkov, Control theory from the geometric viewpoint, Encyclopaedia of Mathematical Sciences 87, Control Theory and Optimization II. Springer-Verlag, Berlin (2004).

[2] F. Alouges, A. DeSimone and A. Lefebvre, Optimal strokes for low Reynolds number swimmers: an example. J. Nonlinear Sci. 18 (2008) 277-302.

[3] H.C. Berg and R. Anderson, Bacteria swim by rotating their flagellar filaments. Nature 245 (1973) 380-382.

[4] J. Blake, A finite model for ciliated micro-organisms. J. Biomech. 6 (1973) 133-140.

[5] C. Brennen, An oscil lating-boundary-layer theory for ciliary propulsion. J. Fluid Mech. 65 (1974) 799-824.

[6] P. Brunovský and C. Lobry, Contrôlabilité Bang Bang, contrôlabilité différentiable, et perturbation des systèmes non linéaires. Ann. Mat. Pura Appl. 105 (1975) 93-119.

[7] S. Childress, Mechanics of swimming and flying, Cambridge Studies in Mathematical Biology 2. Cambridge University Press, Cambridge (1981).

[8] Y. Chitour, J.-M. Coron and M. Garavello, On conditions that prevent steady-state controllability of certain linear partial differential equations. Discrete Contin. Dyn. Syst. 14 (2006) 643-672.

[9] G.P. Galdi, An introduction to the mathematical theory of the Navier-Stokes equations I: Linearized steady problems, Springer Tracts in Natural Philosophy 38. Springer-Verlag, New York (1994)

[10] K.A. Grasse and H.J. Sussmann, Global controllability by nice controls, in Nonlinear controllability and optimal control, Monogr. Textbooks Pure Appl. Math. 133, Dekker, New York (1990) 33-79.

[11] J. Happel and H. Brenner, Low Reynolds number hydrodynamics with special applications to particulate media. Prentice-Hall Inc., Englewood Cliffs, USA (1965).

[12] V. Jurdjevic, Geometric control theory, Cambridge Studies in Advanced Mathematics 52. Cambridge University Press, Cambridge (1997).

[13] V. Jurdjevic and I. Kupka, Control systems subordinated to a group action: accessibility. J. Differ. Equ. 39 (1980) $186-211$.

[14] V. Jurdjevic and I. Kupka, Control systems on semi-simple Lie groups and their homogeneous sapces. Ann. Inst. Fourier 31 (1981) 151-179.

[15] V. Jurdjevic and G. Sallet, Controllability properties of affine systems. SIAM J. Contr. Opt. 22 (1984) 501-508.

[16] S. Keller and T. Wu, A porous prolate-spheroidal model for ciliated micro-organisms. J. Fluid Mech. 80 (1977) $259-278$.

[17] J. Lighthill, Mathematical Biofluiddynamics, Regional Conference Series in Applied Mathematics 17. Society for Industrial and Applied Mathematics, Philadelphia, USA (1975). (Based on the lecture course delivered to the Mathematical Biofluiddynamics Research Conference of the National Science Foundation held from July 16-20 1973, at Rensselaer Polytechnic Institute, Troy, New York, USA.)

[18] E.M. Purcell, Life at low Reynolds numbers. Am. J. Phys. 45 (1977) 3-11.

[19] J. San Martín, T. Takahashi and M. Tucsnak, A control theoretic approach to the swimming of microscopic organisms. Quart. Appl. Math. 65 (2007) 405-424.

[20] J. Simon, Différentiation de problèmes aux limites par rapport au domaine. Lecture notes, University of Seville, Spain (1991).

[21] H.J. Sussmann, Some properties of vector field systems that are not altered by small perturbations. J. Differ. Equ. 20 (1976) 292-315.

[22] G. Taylor, Analysis of the swimming of microscopic organisms. Proc. Roy. Soc. London. Ser. A 209 (1951) $447-461$. 